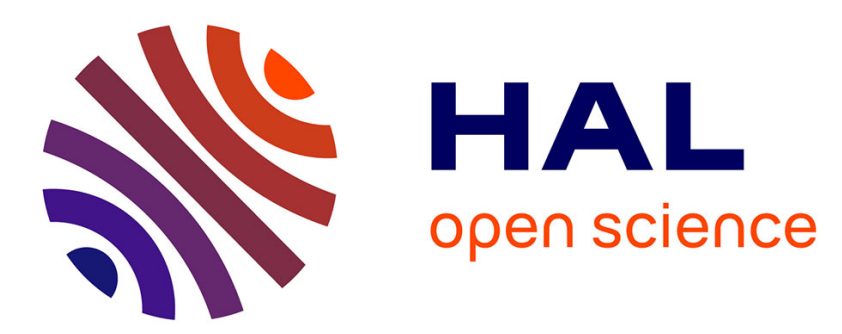

\title{
Study of the mixed finite volume method for Stokes and Navier-Stokes equations
}

\author{
Jerome Droniou, Robert Eymard
}

\section{To cite this version:}

Jerome Droniou, Robert Eymard. Study of the mixed finite volume method for Stokes and NavierStokes equations. Numerical Methods for Partial Differential Equations, 2008, 10.1002/num.20333 . hal-00110911

\section{HAL Id: hal-00110911 \\ https://hal.science/hal-00110911}

Submitted on 2 Nov 2006

HAL is a multi-disciplinary open access archive for the deposit and dissemination of scientific research documents, whether they are published or not. The documents may come from teaching and research institutions in France or abroad, or from public or private research centers.
L'archive ouverte pluridisciplinaire HAL, est destinée au dépôt et à la diffusion de documents scientifiques de niveau recherche, publiés ou non, émanant des établissements d'enseignement et de recherche français ou étrangers, des laboratoires publics ou privés. 


\title{
Study of the mixed finite volume method for Stokes and Navier-Stokes equations
}

\author{
Jérôme Droniou*, Robert Eymard ${ }^{\dagger}$
}

$23 / 10 / 2006$

\begin{abstract}
We present finite volume schemes for Stokes and Navier-Stokes equations. These schemes are based on the mixed finite volume introduced in [6], and can be applied on any type of grid (without "orthogonality" assumptions as for classical finite volume methods) and in any space dimension. We present numerical results on some irregular grids, and we prove, for both Stokes and Navier-Stokes equations, the convergence of the scheme toward a solution of the continuous problem.
\end{abstract}

Keywords. Mixed finite volume scheme, Stokes problem, Navier-Stokes problem, general grids, numerical results.

\section{Introduction}

Finding an approximate solution of the Navier-Stokes equations can be done by a large range of numerical methods: finite element methods, mostly used by the mathematician community (see for example $[10,11,12]$ and references therein), spectral methods and finite volume methods, largely used by the engineering and physicists community (one can first refer to [15] for finite volume methods on staggered grids, and for example to $[2,13,8,9]$ for colocated finite volume schemes). One reason for this difference of practice is that an advantage of finite volume methods on finite element ones lies on its easy physical interpretation and on simpler implementations. However, on domains with complex shapes, it remains difficult to account for constraints provided by finite volume schemes on the meshes: the well-known Patankar scheme on staggered grids can hardly be extended to unstructured meshes, and the implementation of colocated finite volume schemes is complex on general meshes, demanding a stabilization procedure for the pressure.

These constraints on the grids are due to the simultaneous discretization of the viscous term in the momentum balance equation and of the mass conservation equation. On the other hand, a mixed finite volume scheme has recently been shown to be well suited for the resolution of diffusion problems on any type of $2 \mathrm{D}$ or $3 \mathrm{D}$ grid, structured or not, admissible or not in the sense

\footnotetext{
*Département de Mathématiques, UMR CNRS 5149, CC 051, Université Montpellier II, Place Eugène Bataillon, 34095 Montpellier cedex 5, France. email: droniou@math.univ-montp2.fr

${ }^{\dagger}$ Laboratoire d'Analyse et de Mathématiques Appliquées, UMR 8050, Université de Marne-la-Vallée, 5 boulevard Descartes, Champs-sur-Marne, 77454 Marne-la-Vallée Cedex 2, France. email:Robert.Eymard@univ-mlv.fr
} 
of finite element or classical finite volume methods. Hence one could expect that this scheme would provide new gridding possibilities in the case of Stokes and Navier-Stokes equations. This is the point that we intend to make in this paper. Let us first recall the continuous problems that are to be approximated.

We first consider the Stokes problem and we therefore search for an approximation of $\bar{u}=$ $\left(\bar{u}^{(1)}, \ldots, \bar{u}^{(d)}\right)$ and $\bar{p}$, weak solution to

$$
\begin{aligned}
& -\Delta \bar{u}+\nabla \bar{p}=f \quad \text { in } \Omega, \\
& \operatorname{div} \bar{u}=0 \quad \text { in } \Omega, \\
& \bar{u}=0 \quad \text { on } \partial \Omega, \\
& \int_{\Omega} \bar{p}(x) \mathrm{d} x=0,
\end{aligned}
$$

under the following assumptions:

$\Omega$ is an open bounded connected polygonal subset of $\mathbb{R}^{d}, d=2$ or 3 ,

and

$$
f=\left(f^{(1)}, \ldots, f^{(d)}\right) \in L^{2}(\Omega)^{d} .
$$

Thanks to Lax-Milgram theorem, there exists a unique weak solution to (1) in the following sense.

Definition 1.1 [Weak solution to the Stokes equation] Assume that (2) and (3) hold. A weak solution to (1) is $\bar{u}$ such that

$$
\left\{\begin{array}{l}
\bar{u} \in E(\Omega), \\
\int_{\Omega} \nabla \bar{u}(x): \nabla \varphi(x) \mathrm{d} x=\int_{\Omega} f(x) \cdot \varphi(x) \mathrm{d} x, \quad \forall \varphi \in E(\Omega),
\end{array}\right.
$$

where we define $E(\Omega)=\left\{\varphi \in H_{0}^{1}(\Omega)^{d}, \operatorname{div}(\varphi)=0\right\}$ and $\nabla \bar{u}(x): \nabla \varphi(x)=\sum_{i=1}^{d} \nabla \bar{u}^{(i)}(x)$. $\nabla \varphi^{(i)}(x)$.

We also consider the incompressible transient Navier-Stokes problem:

$$
\begin{aligned}
& \left.\partial_{t} \bar{u}+(\bar{u} \cdot \nabla) \bar{u}-\Delta \bar{u}+\nabla \bar{p}=f \quad \text { in }\right] 0, T[\times \Omega, \\
& \operatorname{div} \bar{u}=0 \quad \text { in }] 0, T[\times \Omega, \\
& \bar{u}=0 \quad \text { on }] 0, T[\times \partial \Omega, \\
& \bar{u}(0, \cdot)=u_{0} \quad \text { in } \Omega \\
& \left.\int_{\Omega} \bar{p}(\cdot, x) \mathrm{d} x=0 \quad \text { on }\right] 0, T[,
\end{aligned}
$$

under the assumption

$$
f \in L^{2}(] 0, T[\times \Omega)^{d}, u_{0} \in L^{2}(\Omega)^{d} .
$$


Remark 1.1 [Renormalization] If we replace the first equation of (5) by $\partial_{t} \bar{u}+(\bar{u} \cdot \nabla) \bar{u}-$ $\mu \Delta \bar{u}+\nabla \bar{p}=f$ for some $\mu>0$, then any solution $(\bar{u}(t, x), \bar{p}(t, x))$ of the system of equations thus obtained is such that $\left(\bar{u}(t / \mu, x) / \mu, \bar{p}(t / \mu, x) / \mu^{2}\right)$ is a solution of $(5)$, replacing $u_{0}(x)$ by $u_{0}(x) / \mu$, $f(t, x)$ by $f(t / \mu, x) / \mu^{2}$ and $T$ by $\mu T$. We can therefore let $\mu=1$ in this paper without loss of generality.

It is known $[17,3]$ that there exists a weak solution to $(5)$ in the following sense (notice however that we do not use, in the following, the existence of such a solution).

Definition 1.2 [Weak solution to the Navier-Stokes equation] Assume that (2) and (6) hold. A weak solution to (5) is $\bar{u} \in L^{2}\left(0, T ; H_{0}^{1}(\Omega)\right)^{d}$ such that $\operatorname{div}(\bar{u})=0$ a.e. on $] 0, T[\times \Omega$ and, for all $\varphi \in C_{c}^{\infty}\left(\left[0, T[\times \Omega)^{d}\right.\right.$ such that $\operatorname{div}(\varphi)=0$,

$$
\begin{aligned}
-\int_{0}^{T} \int_{\Omega} \bar{u}(t, x) \cdot \partial_{t} \varphi(t, x) \mathrm{d} t \mathrm{~d} x & +\int_{0}^{T} \int_{\Omega}[(\bar{u}(t, x) \cdot \nabla) \bar{u}(t, x)] \cdot \varphi(t, x) \mathrm{d} t \mathrm{~d} x \\
+\int_{0}^{T} \int_{\Omega} \nabla \bar{u}(t, x): \nabla \varphi(t, x) \mathrm{d} t \mathrm{~d} x & \\
& =\int_{\Omega} u_{0}(x) \cdot \varphi(0, x) \mathrm{d} x+\int_{0}^{T} \int_{\Omega} f(t, x) \cdot \varphi(t, x) \mathrm{d} t \mathrm{~d} x .
\end{aligned}
$$

The principle of our scheme, described in Section 2, is the following. We simultaneously look for approximations $u_{K}^{(i)}, \mathbf{v}_{K}^{(i)}$ of $\bar{u}^{(i)}, \nabla \bar{u}^{(i)}$ in each control volume $K$ and for approximation $\widetilde{F}_{\sigma}^{(i)}$ of $\int_{\sigma} \nabla \bar{u}^{(i)}(x) \cdot \mathbf{n}_{\sigma} \mathrm{d} \gamma(x)$ at each edge $\sigma$ of the mesh, where $\mathbf{n}_{\sigma}$ is a unit vector normal to $\sigma$. The values $\widetilde{F}_{\sigma}^{(i)}$ must then satisfy the balance equation in each control volume, and consistency relations are imposed on $u_{K}^{(i)}, \mathbf{v}_{K}^{(i)}$ and $\widetilde{F}_{\sigma}^{(i)}$. We present some numerical examples in Section 3, which demonstrate the aptitude of the mixed finite volume scheme for providing accurate results on meshes including refinements, vertices inside internal edges and general quadrangular control volumes. In Sections 4 and 5, we study the mixed finite volume approximation respectively for Stokes and Navier-Stokes equations: we show that this method leads to systems (linear in the case of Stokes problem, non-linear in the case of Navier-Stokes problem) which, in general, have at least one approximate solution $u, \mathbf{v}$ and $F$ (this solution is unique in the case of Stokes problem), and we prove the convergence of these approximate solutions toward a solution of the continuous equations. An appendix (Section 6) concludes the paper by giving various lemmas useful for the analysis of the schemes.

\section{The mixed finite volume schemes}

\subsection{Admissible discretization of $\Omega$}

We present the notion of admissible discretization of the domain $\Omega$, which is necessary to give the expression of the finite volume scheme.

Definition 2.1 [Admissible discretization] Let $\Omega$ be an open bounded polygonal subset of $\mathbb{R}^{d}(d \geq 1)$, and $\partial \Omega=\bar{\Omega} \backslash \Omega$ its boundary. An admissible discretization of $\Omega$ is given by $\mathcal{D}=(\mathcal{M}, \mathcal{E}, \mathcal{P})$, where: 
- $\mathcal{M}$ is a finite family of non empty open polygonal disjoint subsets of $\Omega$ (the "control volumes") such that $\bar{\Omega}=\cup_{K \in \mathcal{M}} \bar{K}$.

- $\mathcal{E}$ is a finite family of disjoint subsets of $\bar{\Omega}$ (the "edges" of the mesh), such that, for all $\sigma \in \mathcal{E}$, there exists an affine hyperplane $E$ of $\mathbb{R}^{d}$ and $K \in \mathcal{M}$ with $\sigma \subset \partial K \cap E$ and $\sigma$ is a non empty open subset of $E$. We assume that, for all $K \in \mathcal{M}$, there exists a subset $\mathcal{E}_{K}$ of $\mathcal{E}$ such that $\partial K=\cup_{\sigma \in \mathcal{E}_{K}} \bar{\sigma}$. We also assume that, for all $\sigma \in \mathcal{E}$, either $\sigma \subset \partial \Omega$ or $\sigma \subset \bar{K} \cap \bar{L}$ for some $(K, L) \in \mathcal{M}^{2}$.

- $\mathcal{P}$ is a family of points of $\Omega$ indexed by $\mathcal{M}$, denoted by $\mathcal{P}=\left(\mathbf{x}_{K}\right)_{K \in \mathcal{M}}$ and such that, for all $K \in \mathcal{M}, \mathbf{x}_{K} \in K$.

Remark 2.1 Though the elements of $\mathcal{E}_{K}$ may not be the real edges of a control volume $K$ (each $\sigma \in \mathcal{E}_{K}$ may be only a part of a full edge, especially in the case of locally refined grids), we will in the following call "edges of $K$ " the elements of $\mathcal{E}_{K}$. Notice also that the control volumes can be non-convex, so that two neighboring control volumes can share multiple edges.

Notations. The measure of a control volume $K$ is denoted by $\mathrm{m}(K)$ and the $(d-1)$-dimensional measure of an edge $\sigma$ by $\mathrm{m}(\sigma)$. If $\sigma$ is a given edge, we sometime write it $\sigma^{K \mid L}$ to indicate that the control volumes on each side of $\sigma$ are $K$ and $L$; if $\sigma$ is a boundary edge, $\sigma^{K \mid \partial}$ indicates that the control volume whose boundary contains $\sigma$ is $K$. For all $\sigma \in \mathcal{E}, \mathbf{x}_{\sigma}$ is the barycenter of $\sigma$. The set of interior (resp. boundary) edges is denoted by $\mathcal{E}_{\text {int }}$ (resp. $\mathcal{E}_{\text {ext }}$ ), that is $\mathcal{E}_{\text {int }}=\{\sigma \in \mathcal{E}$; $\sigma \not \subset \partial \Omega\}$ (resp. $\mathcal{E}_{\text {ext }}=\{\sigma \in \mathcal{E} ; \sigma \subset \partial \Omega\}$ ). For all $K \in \mathcal{M}$, we denote by $\mathcal{N}_{K}$ the subset of $\mathcal{M}$ of the neighboring control volumes (that is, the $L$ such that $\bar{K} \cap \bar{L}$ contains an edge of the discretization), and we denote by $\mathcal{E}_{K \text {,ext }}=\mathcal{E}_{K} \cap \mathcal{E}_{\text {ext }}$ and $\mathcal{E}_{K \text {,int }}=\mathcal{E}_{K} \cap \mathcal{E}_{\text {int }}$.

We denote by $H_{\mathcal{D}}$ the set of functions $w: \Omega \rightarrow \mathbb{R}$ which are piecewise constant on each control volume $K \in \mathcal{M}$, and we identify $w \in H_{\mathcal{D}}$ with the family of its values $\left(w_{K}\right)_{K \in \mathcal{M}}$ on the control volumes. $\mathcal{F}_{\mathcal{D}}$ is the set of families of real numbers $\left(F_{K, \sigma}\right)_{K \in \mathcal{M}, \sigma \in \mathcal{E}_{K}}$.

To study the convergence of the schemes, we need the following two quantities: the size of the discretization

$$
\operatorname{size}(\mathcal{D})=\sup \{\operatorname{diam}(K) ; K \in \mathcal{M}\}
$$

and the regularity of the discretization

$$
\begin{aligned}
\operatorname{regul}(\mathcal{D})= & \sup \left\{\max \left(\frac{\operatorname{diam}(K)^{d}}{\rho_{K}^{d}}, \operatorname{Card}\left(\mathcal{E}_{K}\right)\right) ; K \in \mathcal{M}\right\} \\
& +\sup \left\{\frac{\operatorname{diam}(K)}{\operatorname{diam}(L)} ; K \in \mathcal{M}, L \in \mathcal{N}_{K}\right\}
\end{aligned}
$$

where, for $K \in \mathcal{M}, \rho_{K}$ is the supremum of the real numbers $r>0$ such that $K$ is star-shaped with respect to all the points in a ball of radius $r$. Notice in particular that, for all $K \in \mathcal{M}$, $\operatorname{diam}(K)^{d} \leq \operatorname{regul}(\mathcal{D}) \rho_{K}^{d} \leq \frac{\operatorname{regul}(\mathcal{D})}{\omega_{d}} \mathrm{~m}(K)$ (with $\omega_{d}$ the volume of the unit ball in $\mathbb{R}^{d}$ ); hence, since $\operatorname{Card}\left(\mathcal{E}_{K}\right) \leq \operatorname{regul}(\mathcal{D})$ and $\mathrm{m}(\sigma) \leq \omega_{d-1} \operatorname{diam}(K)^{d-1}$ if $\sigma \in \mathcal{E}_{K}$, we have

$$
\sum_{\sigma \in \mathcal{E}_{K}} \mathrm{~m}(\sigma) \operatorname{diam}(K) \leq \frac{\omega_{d-1} \operatorname{regul}(\mathcal{D})^{2}}{\omega_{d}} \mathrm{~m}(K) .
$$

Note also that $\operatorname{regul}(\mathcal{D})$ does not increase in a local refinement procedure, provided that this refinement is built by layers. 


\subsection{A mixed finite volume scheme for Stokes problem}

Let $\mathcal{D}$ be an admissible discretization of $\Omega$. Taking $\nu>0$, the numerical scheme for the Stokes problem is the following: find $p \in H_{\mathcal{D}}$ and, for all $i=1, \ldots, d,\left(u^{(i)}, \mathbf{v}^{(i)}, \widetilde{F}^{(i)}\right) \in H_{\mathcal{D}} \times H_{\mathcal{D}}^{d} \times \mathcal{F}_{\mathcal{D}}$ which satisfies the following equations. The first relation states that $\mathbf{v}^{(i)}$ is the gradient of $u^{(i)}$ (we penalize with the fluxes of $\mathbf{v}^{(i)}$ in order to estimate them later on):

$$
\begin{array}{r}
\mathbf{v}_{K}^{(i)} \cdot\left(\mathbf{x}_{\sigma}-\mathbf{x}_{K}\right)+\mathbf{v}_{L}^{(i)} \cdot\left(\mathbf{x}_{L}-\mathbf{x}_{\sigma}\right)+\nu \frac{\operatorname{diam}(K)}{\mathrm{m}(\sigma)} \widetilde{F}_{K, \sigma}^{(i)}-\nu \frac{\operatorname{diam}(L)}{\mathrm{m}(\sigma)} \widetilde{F}_{L, \sigma}^{(i)}=u_{L}^{(i)}-u_{K}^{(i)}, \\
\forall \sigma^{K \mid L} \in \mathcal{E}_{\text {int }}, \forall i=1, \ldots, d, \\
\mathbf{v}_{K}^{(i)} \cdot\left(\mathbf{x}_{\sigma}-\mathbf{x}_{K}\right)+\nu \frac{\operatorname{diam}(K)}{\mathrm{m}(\sigma)} \widetilde{F}_{K, \sigma}^{(i)}=-u_{K}^{(i)}, \quad \forall \sigma^{K \mid \partial} \in \mathcal{E}_{\text {ext }}, \forall i=1, \ldots, d .
\end{array}
$$

We then introduce "complete fluxes", which gather the fluxes of $\mathbf{v}^{(i)}$ and of the pressure (these are the fluxes involved in the first P.D.E. of (1)), and we ask for them to be conservative:

$$
\begin{gathered}
F_{K, \sigma}^{(i)}=\widetilde{F}_{K, \sigma}^{(i)}-p_{K} \operatorname{m}(\sigma) \mathbf{e}^{(i)} \cdot \mathbf{n}_{K, \sigma}, \quad \forall K \in \mathcal{M}, \forall \sigma \in \mathcal{E}_{K}, \forall i=1, \ldots, d, \\
F_{K, \sigma}^{(i)}+F_{L, \sigma}^{(i)}=0, \quad \forall \sigma^{K \mid L} \in \mathcal{E}_{\text {int }}, \forall i=1, \ldots, d
\end{gathered}
$$

$\left(\mathbf{e}^{(1)}, \ldots, \mathbf{e}^{(d)}\right.$ is the canonical basis of $\left.\mathbb{R}^{d}\right)$. The link between $\mathbf{v}^{(i)}$ and its fluxes $\widetilde{F}^{(i)}$ is that the latter allows to reconstruct the former (see Lemma 6.1):

$$
\mathrm{m}(K) \mathbf{v}_{K}^{(i)}=\sum_{\sigma \in \mathcal{E}_{K}} \widetilde{F}_{K, \sigma}^{(i)}\left(\mathbf{x}_{\sigma}-\mathbf{x}_{K}\right), \quad \forall K \in \mathcal{M}, \forall i=1, \ldots, d .
$$

The following equation translates the incompressibility condition, taking into account the penalization introduced in (9):

$$
\mathrm{m}(K) \sum_{i=1}^{d} \mathbf{v}_{K}^{(i)} \cdot \mathbf{e}^{(i)}+\nu \operatorname{diam}(K) \sum_{i=1}^{d} \sum_{\sigma \in \mathcal{E}_{K}} \widetilde{F}_{K, \sigma}^{(i)} \mathbf{e}^{(i)} \cdot \mathbf{n}_{K, \sigma}=0, \quad \forall K \in \mathcal{M} .
$$

We then write the balance of fluxes, that is to say the discrete counterpart of the integration of the first P.D.E. in (1) on each control volume:

$$
-\sum_{\sigma \in \mathcal{E}_{K}} F_{K, \sigma}^{(i)}=\int_{K} f^{(i)}(x) \mathrm{d} x, \quad \forall K \in \mathcal{M}, \forall i=1, \ldots, d,
$$

and we normalize the choice of the pressure:

$$
\sum_{K \in \mathcal{M}} \mathrm{m}(K) p_{K}=0
$$

Remark 2.2 [Square system] A close examination of the preceding scheme shows that it is over-determined. Indeed, by (10) and (11) there is in fact only one unknown flux $\widetilde{F}_{\sigma}^{(i)}$ for each edge of the mesh (and each $i=1, \ldots, d$ ) - since the knowledge of $\widetilde{F}_{K, \sigma}^{(i)}$ gives back $\widetilde{F}_{L, \sigma}^{(i)}$ using $p_{K}$ and $p_{L}$ - and (9) precisely provides one equation per edge (for each $\left.i=1, \ldots, d\right)$; (12) and (14) respectively give as many equations as there are unknowns $\mathbf{v}_{K}^{(i)}$ and $u_{K}^{(i)}$, and (13) gives as 
many equations as the unknowns $p_{K}$. With (15), we therefore have written one more equation than we have unknowns.

However, these equations are not free: multiply each equation (9) by $\mathrm{m}(\sigma) \mathbf{e}^{(i)} \cdot \mathbf{n}_{K, \sigma}$, sum on $i=1, \ldots, d$ and on $\sigma \in \mathcal{E}$. Gathering by control volumes, Lemma 6.1 gives

$$
\begin{aligned}
\sum_{K \in \mathcal{M}} \mathrm{m}(K) \sum_{i=1}^{d} \mathbf{v}_{K}^{(i)} \cdot \mathbf{e}^{(i)}+\sum_{K \in \mathcal{M}} \nu & \operatorname{diam}(K) \sum_{i=1}^{d} \sum_{\sigma \in \mathcal{E}_{K}} \widetilde{F}_{K, \sigma}^{(i)} \mathbf{e}^{(i)} \cdot \mathbf{n}_{K, \sigma} \\
& =-\sum_{i=1}^{d} \sum_{K \in \mathcal{M}} u_{K}^{(i)} \mathbf{e}^{(i)} \sum_{\sigma \in \mathcal{E}_{K}} \mathrm{~m}(\sigma) \mathbf{n}_{K, \sigma} .
\end{aligned}
$$

Since $\sum_{\sigma \in \mathcal{E}_{K}} \mathrm{~m}(\sigma) \mathbf{n}_{K, \sigma}=0$ (thanks to Stokes' formula), the right-hand side of this equation is null, and (13) shows that the left-hand side is also null. Hence, equations (9) and (13) are linked in a non-trivial fashion, and we can in fact suppress one of the equations in (13) (for example) and get back a square system, equivalent to (9)-(15).

Remark 2.3 [Exact incompressibility] The equations (9) allow to define $u_{\sigma}^{(i)}$ by

$$
\mathbf{v}_{K}^{(i)} \cdot\left(\mathbf{x}_{\sigma}-\mathbf{x}_{K}\right)+\nu \frac{\operatorname{diam}(K)}{\mathrm{m}(\sigma)} \widetilde{F}_{K, \sigma}^{(i)}=u_{\sigma}^{(i)}-u_{K}^{(i)}, \quad \forall K \in \mathcal{M}, \forall \sigma \in \mathcal{E}_{K}, \forall i=1, \ldots, d
$$

(and $u_{\sigma}^{(i)}$ indeed only depends on $\sigma$, not on $K$ such that $\sigma \in \mathcal{E}_{K}$ ). Multiplying these equations by $\mathrm{m}(\sigma) \mathbf{e}^{(i)} \cdot \mathbf{n}_{K, \sigma}$ and summing on $\sigma \in \mathcal{E}_{K}$, we obtain, thanks to Lemma 6.1 and since $\sum_{\sigma \in \mathcal{E}_{K}} \mathrm{~m}(\sigma) \mathbf{e}^{(i)} \cdot \mathbf{n}_{K, \sigma}=0$

$$
\mathrm{m}(K) \mathbf{v}_{K}^{(i)} \cdot \mathbf{e}^{(i)}+\nu \operatorname{diam}(K) \sum_{\sigma \in \mathcal{E}_{K}} \widetilde{F}_{K, \sigma}^{(i)} \mathbf{e}^{(i)} \cdot \mathbf{n}_{K, \sigma}=\sum_{\sigma \in \mathcal{E}_{K}} u_{\sigma}^{(i)} \mathrm{m}(\sigma) \mathbf{e}^{(i)} \cdot \mathbf{n}_{K, \sigma} .
$$

If we define $\vec{u}_{\sigma}=\left(u_{\sigma}^{(1)}, \ldots, u_{\sigma}^{(d)}\right)$, summing the preceding equations on $i=1, \ldots, d$ and using (13) leads to

$$
\sum_{\sigma \in \mathcal{E}_{K}} \mathrm{~m}(\sigma) \vec{u}_{\sigma} \cdot \mathbf{n}_{K, \sigma}=0
$$

The scheme we present is therefore indeed a finite volume method.

\subsection{A mixed finite volume scheme for transient Navier-Stokes problem}

Assume (2) and let $T>0$. If $\mathcal{D}$ is an admissible discretization of $\Omega$ in the sense of Definition 2.1 and $\delta t>0$ is such that $N_{\delta t}=T / \delta t$ is a positive integer, we denote $H_{\mathcal{D}, \delta t}$ the families of real numbers $w=\left(w_{K}^{n+1 / 2}\right)_{K \in \mathcal{M}, n=0, \ldots, N_{\delta t}-1}$, and we identify $w \in H_{\mathcal{D}, \delta t}$ with the piecewise constant function $w:] 0, T\left[\times \Omega \rightarrow \mathbb{R}\right.$ which is equal to $w_{K}^{n+1 / 2}$ on $] n \delta t,(n+1) \delta t\left[\times K\left(\right.\right.$ for $n=0, \ldots, N_{\delta t}-1$ and $K \in \mathcal{M})$. If $w \in H_{\mathcal{D}, \delta t}$, we denote $w^{n+1 / 2}=\left(w_{K}^{n+1 / 2}\right)_{K \in \mathcal{M}} \in H_{\mathcal{D}}$.

Defining $\mathcal{F}_{\mathcal{D}, \delta t}=\left\{\left(G^{n+1 / 2}\right)_{n=0, \ldots, N_{\delta t}-1} ; \forall n=0, \ldots, N_{\delta t}-1, G^{n+1 / 2} \in \mathcal{F}_{\mathcal{D}}\right\}$, the mixed finite volume scheme for the transient Navier-Stokes problem is a natural generalization of the scheme for the Stokes problem, using a Crank-Nicolson discretization of the time derivative (hence the natural exponent $n+1 / 2$, since this time discretization uses quantities at half time steps). We search $p \in H_{\mathcal{D}, \delta t}$ and, for all $i=1, \ldots, d,\left(u^{(i)}, \mathbf{v}^{(i)}, \widetilde{F}^{(i)}\right) \in H_{\mathcal{D}, \delta t} \times H_{\mathcal{D}, \delta t}^{d} \times \mathcal{F}_{\mathcal{D}, \delta t}$ such that, for all $n=0, \ldots, N_{\delta t}-1$, 
- $\mathbf{v}^{(i)}$ plays the role of a gradient of $u^{(i)}$ :

$$
\begin{array}{r}
\left(\mathbf{v}^{(i)}\right)_{K}^{n+1 / 2} \cdot\left(\mathbf{x}_{\sigma}-\mathbf{x}_{K}\right)+\left(\mathbf{v}^{(i)}\right)_{L}^{n+1 / 2} \cdot\left(\mathbf{x}_{L}-\mathbf{x}_{\sigma}\right)+\nu \frac{\operatorname{diam}(K)}{\mathrm{m}(\sigma)}\left(\widetilde{F}^{(i)}\right)_{K, \sigma}^{n+1 / 2} \\
-\nu \frac{\operatorname{diam}(L)}{\mathrm{m}(\sigma)}\left(\widetilde{F}^{(i)}\right)_{L, \sigma}^{n+1 / 2}=\left(u^{(i)}\right)_{L}^{n+1 / 2}-\left(u^{(i)}\right)_{K}^{n+1 / 2}, \\
\forall \sigma^{K \mid L} \in \mathcal{E}_{\text {int }}, \forall i=1, \ldots, d, \\
\left(\mathbf{v}^{(i)}\right)_{K}^{n+1 / 2} \cdot\left(\mathbf{x}_{\sigma}-\mathbf{x}_{K}\right)+\nu \frac{\operatorname{diam}(K)}{\mathrm{m}(\sigma)}\left(\widetilde{F}^{(i)}\right)_{K, \sigma}^{n+1 / 2}=-\left(u^{(i)}\right)_{K}^{n+1 / 2}, \\
\forall \sigma^{K \mid \partial} \in \mathcal{E}_{\text {ext }}, \forall i=1, \ldots, d,
\end{array}
$$

- the complete fluxes, involving the pressure, are conservative:

$$
\begin{array}{r}
\left(F^{(i)}\right)_{K, \sigma}^{n+1 / 2}=\left(\widetilde{F}^{(i)}\right)_{K, \sigma}^{n+1 / 2}-p_{K}^{n+1 / 2} \mathrm{~m}(\sigma) \mathbf{e}^{(i)} \cdot \mathbf{n}_{K, \sigma}, \forall K \in \mathcal{M}, \forall \sigma \in \mathcal{E}_{K}, \\
\forall i=1, \ldots, d, \\
\left(F^{(i)}\right)_{K, \sigma}^{n+1 / 2}+\left(F^{(i)}\right)_{L, \sigma}^{n+1 / 2}=0, \quad \forall \sigma^{K \mid L} \in \mathcal{E}_{\text {int }}, \forall i=1, \ldots, d,
\end{array}
$$

- $\mathbf{v}^{(i)}$ can be reconstructed from its fluxes:

$$
\mathrm{m}(K)\left(\mathbf{v}^{(i)}\right)_{K}^{n+1 / 2}=\sum_{\sigma \in \mathcal{E}_{K}}\left(\widetilde{F}^{(i)}\right)_{K, \sigma}^{n+1 / 2}\left(\mathbf{x}_{\sigma}-\mathbf{x}_{K}\right), \quad \forall K \in \mathcal{M}, \forall i=1, \ldots, d,
$$

- the incompressibility condition holds:

$$
\begin{array}{r}
\mathrm{m}(K) \sum_{i=1}^{d}\left(\mathbf{v}^{(i)}\right)_{K}^{n+1 / 2} \cdot \mathbf{e}^{(i)}+\nu \operatorname{diam}(K) \sum_{i=1}^{d} \sum_{\sigma \in \mathcal{E}_{K}}\left(\widetilde{F}^{(i)}\right)_{K, \sigma}^{n+1 / 2} \mathbf{e}^{(i)} \cdot \mathbf{n}_{K, \sigma}=0, \\
\forall K \in \mathcal{M},
\end{array}
$$

- the PDE is satisfied on the discrete level $\left(^{1}\right)$ :

$$
\begin{aligned}
& \mathrm{m}(K) \frac{\left(u^{(i)}\right)_{K}^{n+1}-\left(u^{(i)}\right)_{K}^{n}}{\delta t}+\sum_{\sigma \in \mathcal{E}_{K}} \mathrm{~m}(\sigma) \vec{u}_{\sigma}^{n+1 / 2} \cdot \mathbf{n}_{K, \sigma}\left(\frac{\left(u^{(i)}\right)_{K}^{n+1 / 2}+\left(u^{(i)}\right)_{L}^{n+1 / 2}}{2}\right) \\
& -\sum_{\sigma \in \mathcal{E}_{K}}\left(F^{(i)}\right)_{K, \sigma}^{n+1 / 2}=\frac{1}{\delta t} \int_{n \delta t}^{(n+1) \delta t} \int_{K} f^{(i)}(t, x) \mathrm{d} t \mathrm{~d} x, \quad \forall K \in \mathcal{M}, \forall i=1, \ldots, d,
\end{aligned}
$$

where the values at full time steps and half time steps are linked in the following way:

$$
\left(u^{(i)}\right)_{K}^{n+1 / 2}=\frac{\left(u^{(i)}\right)_{K}^{n+1}+\left(u^{(i)}\right)_{K}^{n}}{2}, \quad \forall K \in \mathcal{M}, \forall i=1, \ldots, d,
$$

and where, as in Remark 2.3, we define $\vec{u}_{\sigma}^{n+1 / 2}=\left(\left(u^{(1)}\right)_{\sigma}^{n+1 / 2}, \ldots,\left(u^{(d)}\right)_{\sigma}^{n+1 / 2}\right)$ by $\left(^{2}\right)$

$$
\begin{array}{r}
\left(\mathbf{v}^{(i)}\right)_{K}^{n+1 / 2} \cdot\left(\mathbf{x}_{\sigma}-\mathbf{x}_{K}\right)+\nu \frac{\operatorname{diam}(K)}{\mathrm{m}(\sigma)}\left(\widetilde{F}^{(i)}\right)_{K, \sigma}^{n+1 / 2}=\left(u^{(i)}\right)_{\sigma}^{n+1 / 2}-\left(u^{(i)}\right)_{K}^{n+1 / 2}, \\
\forall K \in \mathcal{M}, \forall \sigma \in \mathcal{E}_{K}, \forall i=1, \ldots, d,
\end{array}
$$

\footnotetext{
${ }^{1}$ In the first sum on the edges, we let $L$ be the neighboring control volume of $K$ on the other side of $\sigma$, if $\sigma \in \mathcal{E}_{K, \text { int }}$, or we let $\left(u^{(i)}\right)_{L}^{n+1 / 2}=0$, if $\sigma \in \mathcal{E}_{K, \text { ext }}$.

${ }^{2}$ This definition makes sense thanks to $(16)$.
} 
- the initial condition is discretized as:

$$
\left(u^{(i)}\right)_{K}^{0}=\frac{1}{\mathrm{~m}(K)} \int_{K} u_{0}^{(i)}(x) \mathrm{d} x, \quad \forall K \in \mathcal{M}, \forall i=1, \ldots, d,
$$

- and the choice of the pressure is normalized:

$$
\sum_{K \in \mathcal{M}} \mathrm{m}(K) p_{K}^{n+1 / 2}=0
$$

It will be useful to notice that, thanks to $(23),(20)$ and as in Remark 2.3, we have

$$
\sum_{\sigma \in \mathcal{E}_{K}} \mathrm{~m}(\sigma) \vec{u}_{\sigma}^{n+1 / 2} \cdot \mathbf{n}_{K, \sigma}=0, \quad \forall K \in \mathcal{M}, \forall n=0, \ldots, N_{\delta t}-1
$$

Remark 2.4 [Scheme for the steady problem] We could of course write a scheme for the steady Navier-Stokes problem; this scheme would satisfy the same mathematical results as in Section 5 (the study of the steady Navier-Stokes equations can be easily deduced from that of the transient Navier-Stokes equations).

Remark 2.5 [Implicit discretization] All the mathematical results presented in this paper hold for the $\theta$-scheme, which consists in replacing (22) by $\left(u^{(i)}\right)_{K}^{n+1 / 2}=\theta\left(u^{(i)}\right)_{K}^{n+1}+(1-\theta)\left(u^{(i)}\right)_{K}^{n}$, with $\theta \in[1 / 2,1]$ (the implicit discretization is obtained for $\theta=1$, the Crank-Nicolson discretization being given by $\theta=1 / 2)$. The crucial point is that, in the course of the proof of Proposition 5.1, the new term $\left(\theta-\frac{1}{2}\right) \sum_{i=1}^{d} \sum_{n=0}^{N-1} \sum_{K \in \mathcal{M}} \mathrm{m}(K)\left(\left(u^{(i)}\right)_{K}^{n+1}-\left(u^{(i)}\right)_{K}^{n}\right)^{2}$ appearing in $T_{2}$ is non-negative for $\theta \in[1 / 2,1]$.

\section{Numerical results}

Since this paper is focused on the presentation of the scheme and on the proof of its convergence, we have no room to develop here a thorough comparison between its results and the ones of other schemes. We therefore limit the presentation of numerical results to the illustration of the aptitude of the scheme for handling various types of grids, in the case of steady and transient Navier-Stokes problems, while preserving good qualitative properties on the solution. The resolution of equations (16) - (25) has been implemented in a prototype code written in FORTRAN, and the resolution procedure at each time step is based on under-relaxed Newtonian iterations coupling all the equations (after elimination of $\mathbf{v}$ thanks to (19)). The resulting linear systems are solved by a direct method (Gaussian elimination) or an iterative method (BICGSTAB solver with an ILU preconditioner, see for example [16]). The implementation of the steady problem is identical to that of a time step of the transient problem, without the term resulting from the time derivative, and the steady solution is therefore directly obtained (there is no need to approximate this solution by a transient one).

\subsection{Lid driven cavity}

We first focus on the classical lid driven cavity example with $R e=1000$. Figure 1 shows the results obtained thanks to the scheme (16) - (25) (using a nonhomogeneous boundary condition 

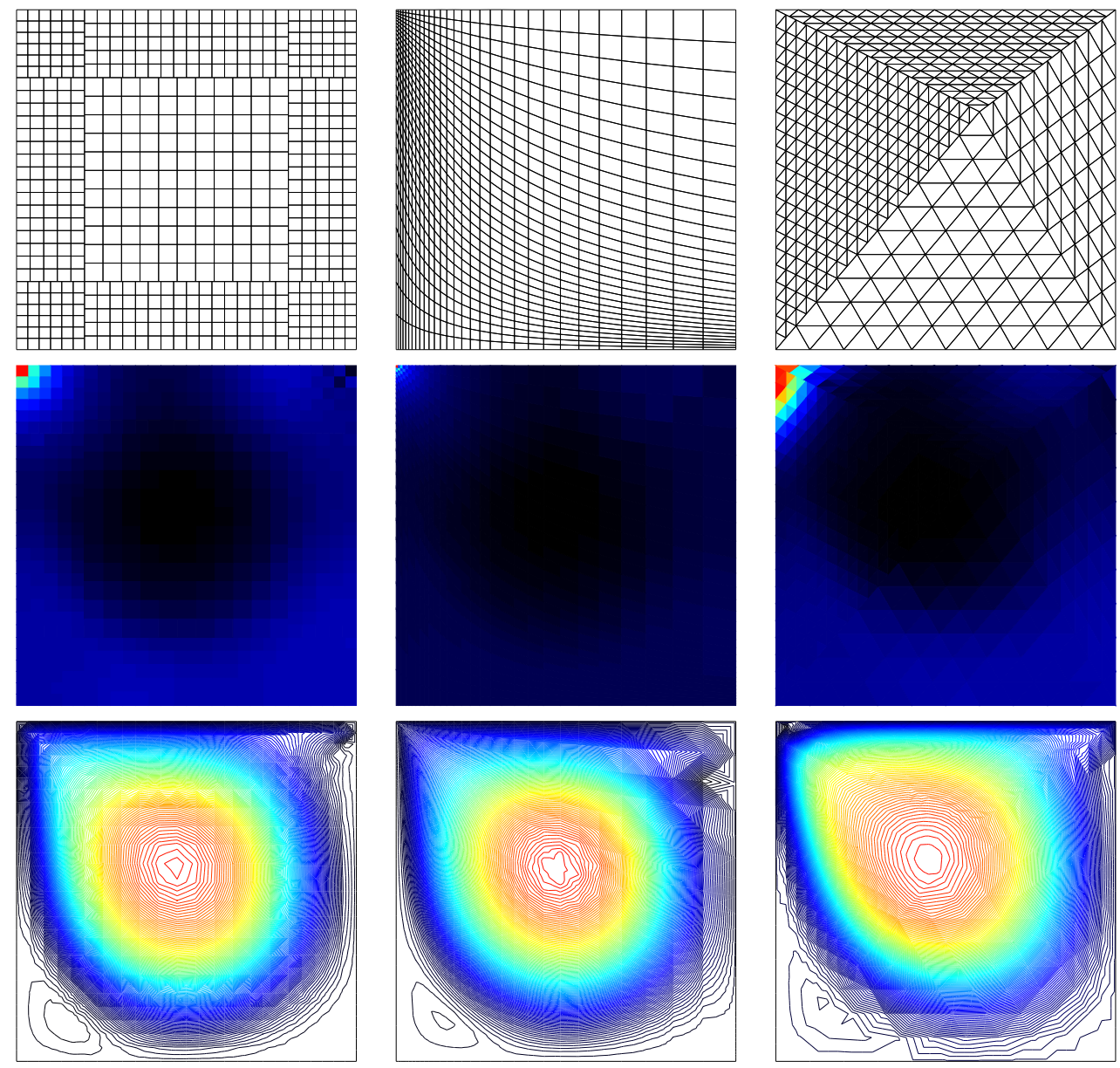

Figure 1: Lid driven cavity on unstructured and irregular grids: grids (top pictures), pressure field (middle pictures) and streamlines (bottom pictures).

instead of the homogeneous one) on different grids which are not admissible for classical finite element or finite volume schemes. The accuracy of these results on those coarse grids appear to be acceptable. We also notice that the quality of the numerical streamlines is mainly linked with the size of the control volumes (the streamlines are deformed in regions with large control volumes, and good in regions with small control volumes), and not with the fact that different regions are discretized with grids which are connected in a "non-admissible" way (in the sense of finite element methods); such a situation can occur, for instance, during a refinement procedure. We present in Figure 2 the effect of the value of the stabilization parameter $\nu$, in the case of the lid driven cavity with $\mathrm{Re}=1000$ on a $30 \times 30$ square grid; these results show that, in order to obtain a good approximate pressure field in this case, one must choose the stabilization parameter not too small. We however want to emphasize that the choice of $\nu$ has no perceptible influence on the quality of the velocity: we have noticed that, on the same $30 \times 30$ grid, the results for the streamlines and the velocity field with $\nu$ in the range $\left[10^{-3}, 10^{-7}\right]$ are indistinguishable from the case $\nu=10^{-7}$ presented in Figure 3 . 

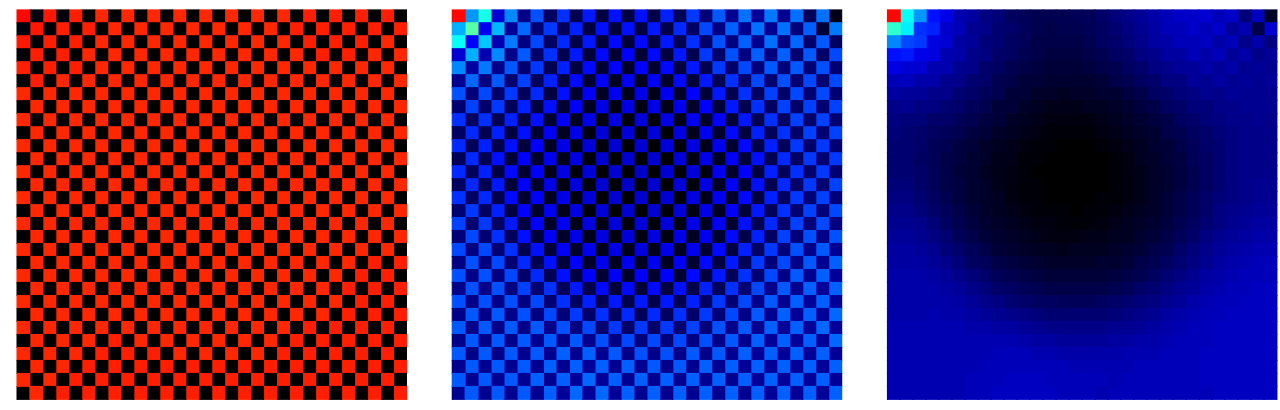

Figure 2: Lid driven cavity on a $30 \times 30$ square grid, pressure fields for: $\nu=10^{-7}$ (left), $\nu=10^{-5}$ (middle), $\nu=10^{-3}$ (right).
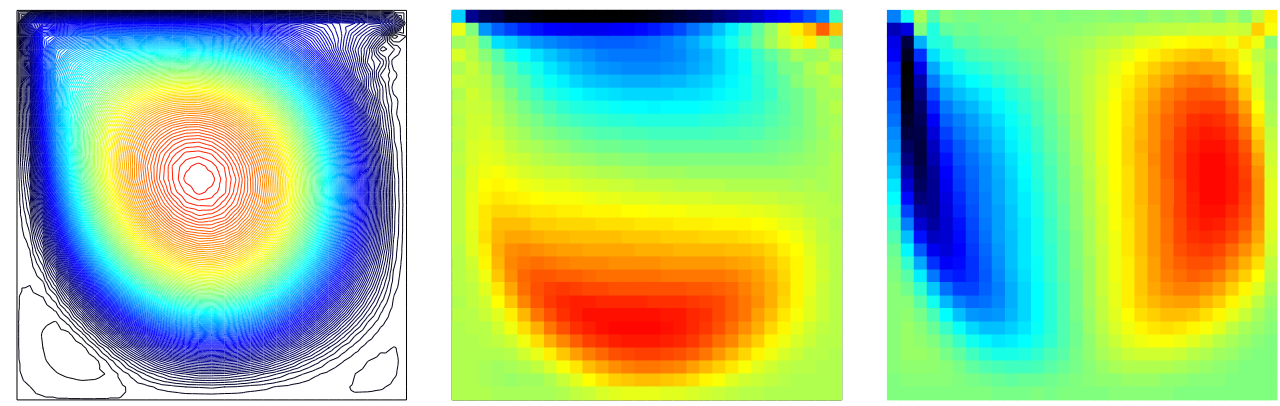

Figure 3: Lid driven cavity on a $30 \times 30$ square grid for $\nu=10^{-7}$ : streamlines (left), horizontal velocity (middle) and vertical velocity (right). 


\subsection{Backward facing step}

We then study the behaviour of the scheme in the case of the flow into a pipe whose dimensions vary discontinuously (the backward facing step problem, included in the domain ] $-2,30[\times] 0,1.94[$, the step being at $x=0$; see for example [1]). We let $\operatorname{Re}=800$ and we use a quite coarse mesh, made of 5625 rectangles and triangles and deliberately chosen to be non admissible in the sense of classical finite element or finite volume schemes (some edges are cut in two, see Figure 4). The results we obtain show a good accuracy: the reattachment length for the bottom vortex is obtained at $x=10.5$, the detachment position for the top vortex is obtained at $x=9.0$ and its reattachment position is given by $x=17.5$, which is in the order of magnitude of the values supplied in the literature, up to 10\% (see Figure 5). Let us also observe that in this case, where we impose the pressure at the right vertical boundary, nearly no stabilization is necessary: we let $\nu=10^{-7}$ for this calculation and we obtain a good pressure field (see Figure 6). Notice finally that, as in the lid driven cavity case, the quality of the numerical results is not deteriorated in the region where the grid is not admissible (in the sense of classical finite element or finite volume).
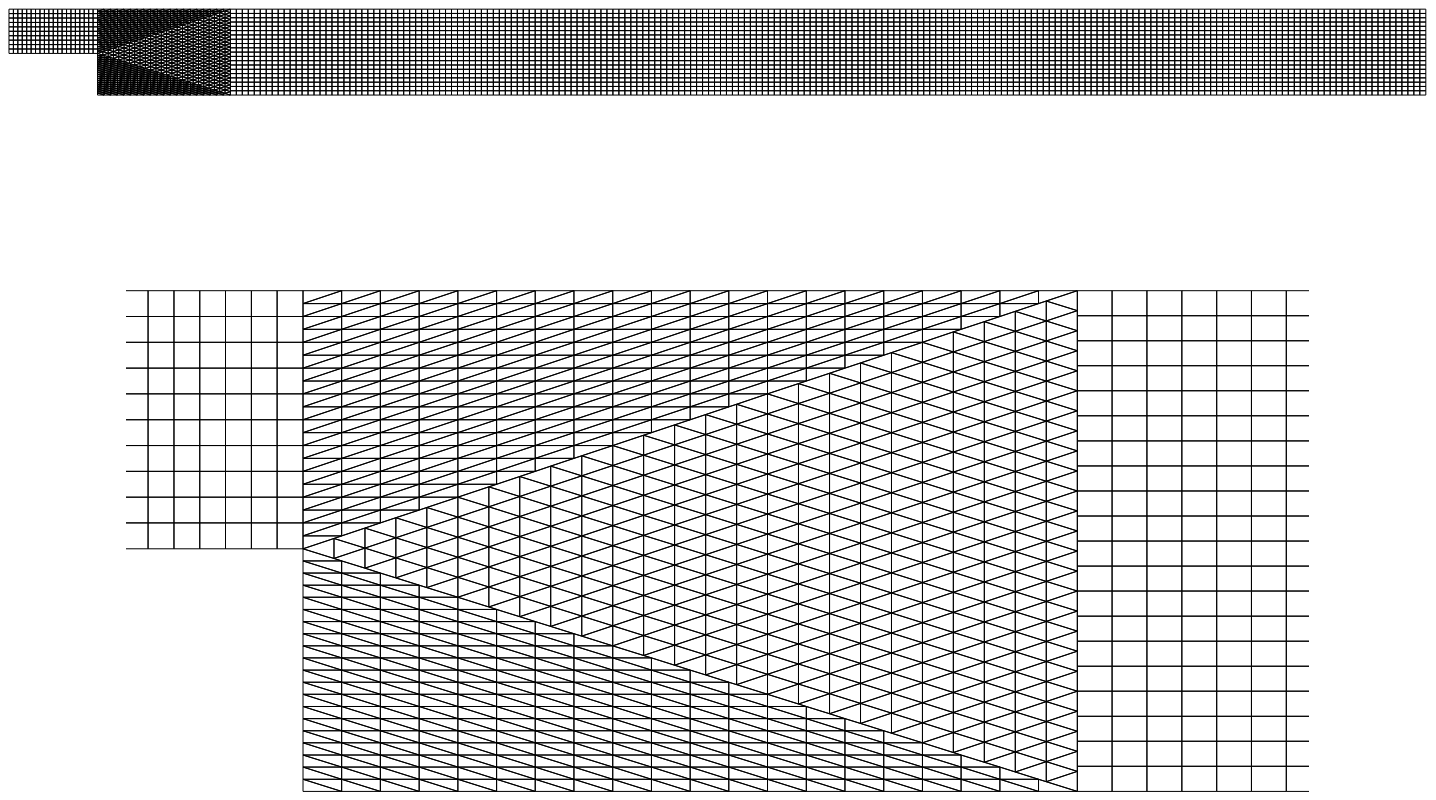

Figure 4: Backward facing step, mesh: in the full pipe (top), and zoom on a neighborhood of the step (bottom).

\subsection{Green-Taylor analytical example}

Let $\mu \in] 0, T[$ be given, and let the pair of functions $(\bar{u}, \bar{p})$ be defined on $] 0, T[\times \Omega$, with $\Omega=$ ] $0,1[\times] 0,1[$ and $T=0.02$, by

$$
\begin{aligned}
& \bar{u}^{(1)}(t, x)=-\frac{1}{\mu} \cos \left(2 \pi\left(x^{(1)}+\frac{1}{4}\right)\right) \sin \left(2 \pi\left(x^{(2)}+\frac{1}{2}\right)\right) \exp \left(-8 \pi^{2} t\right) \\
& \bar{u}^{(2)}(t, x)=\frac{1}{\mu} \sin \left(2 \pi\left(x^{(1)}+\frac{1}{4}\right)\right) \cos \left(2 \pi\left(x^{(2)}+\frac{1}{2}\right)\right) \exp \left(-8 \pi^{2} t\right) \\
& \bar{p}(t, x)=-\frac{1}{4 \mu^{2}}\left(\cos \left(4 \pi\left(x^{(1)}+\frac{1}{4}\right)\right)+\cos \left(4 \pi\left(x^{(2)}+\frac{1}{2}\right)\right)\right) \exp \left(-16 \pi^{2} t\right) .
\end{aligned}
$$



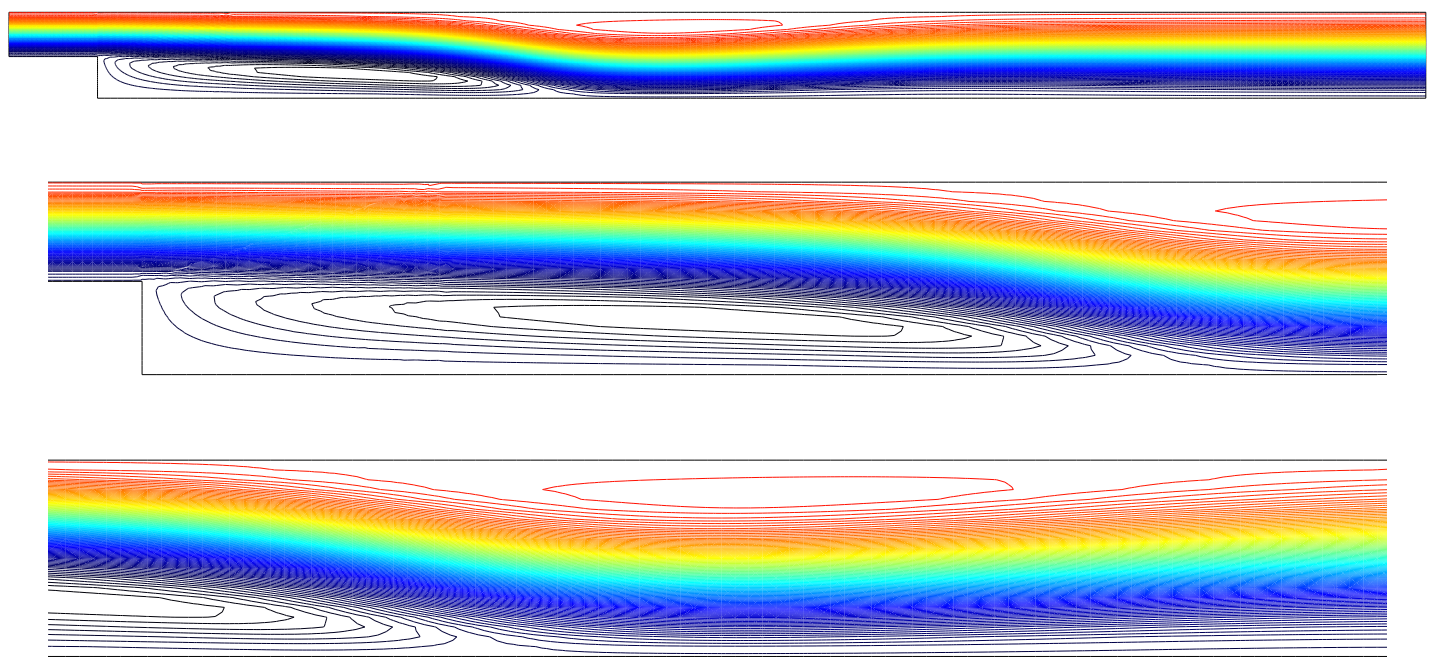

Figure 5: Backward facing step, streamlines: full pipe (top) and zooms on the first (middle) and second (bottom) vortices.

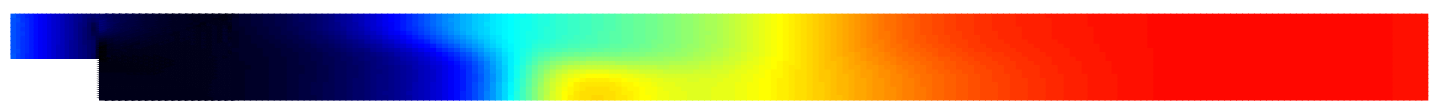

Figure 6: Backward facing step, pressure field.

Then $(\bar{u}, \bar{p})$ is the unique solution of the transient Navier-Stokes equations with $f=0$, the initial condition and nonhomogeneous boundary conditions being respectively given by $\bar{u}(0, \cdot)$ and $\bar{u}(t, x)$ for all $(t, x) \in] 0, T[\times \partial \Omega$ (a small time $T=0.02$ has been selected in order to take into account the exponential decay of the solution: for larger times, the solution nearly vanishes). We denote by $(u, p)$ the velocity and pressure fields resulting from the time implicit version of (16) - (25) (see Remark 2.5; the results given by the Crank-Nicolson scheme have led in this case to lower convergence properties) with $f=0$, the initial condition and the nonhomogeneous boundary conditions satisfied by the continuous solution. The obtained results are given in table 1 (in which the regular grids and the time steps we used are precised), assuming $\mu=0.01$ and setting $\nu=10^{-7}$ for all calculations. These results show that the convergence properties of the

\begin{tabular}{|c|c|c|c|c|}
\hline grid & $\delta t$ & $\frac{\left\|\bar{u}^{(1)}(T, \cdot)-u^{(1)}(T, \cdot)\right\|_{L^{2}}}{\left\|\bar{u}^{(1)}(T, \cdot)\right\|_{L^{2}}}$ & $\frac{\left\|\bar{u}^{(2)}(T, \cdot)-u^{(2)}(T, \cdot)\right\|_{L^{2}}}{\left\|\bar{u}^{(2)}(T, \cdot)\right\|_{L^{2}}}$ & $\frac{\|\bar{p}(T, \cdot)-p(T, \cdot)\|_{L^{2}}}{\|\bar{p}(T, \cdot)\|_{L^{2}}}$ \\
\hline $10 \times 10$ & 0.004 & 0.14 & 0.15 & 0.38 \\
\hline $20 \times 20$ & 0.001 & 0.038 & 0.043 & 0.086 \\
\hline $40 \times 40$ & 0.00025 & 0.011 & 0.012 & 0.023 \\
\hline $80 \times 80$ & 0.0000625 & 0.0029 & 0.0035 & 0.0064 \\
\hline
\end{tabular}

Table 1: Green-Taylor analytical example, relative errors of the different fields of unknowns at time $T=0.02$.

method are compatible with space order not far from 2 and time order not far from 1 for the 
velocity and the pressure, although we let $\nu$ be constant.

\subsection{Conservation of kinetics energy}

It has been noticed that, in order to obtain a stable and dissipation-free numerical method, one of the important behaviors of the scheme must be the conservation, at very high Reynolds numbers and with null source term, of the kinetics energy (see [14]). Another way to say that is that, for Reynolds number equal to 1 and small times (see the renormalization in Remark 1.1), the decay of kinetics energy should only come from the viscous term, not the convective nonlinear one. Let us check the behavior of the mixed finite volume method on the kinetics energy.

We consider (5) with $\Omega=] 0,1[\times] 0,1\left[, T=10^{-5}, f=0\right.$ and $u_{0}$ given by

$$
\begin{array}{ll}
u_{0}^{(1)}\left(x^{(1)}, x^{(2)}\right) & =-\partial_{2} \Psi\left(x^{(1)}, x^{(2)}\right), \\
u_{0}^{(2)}\left(x^{(1)}, x^{(2)}\right) & =\partial_{1} \Psi\left(x^{(1)}, x^{(2)}\right), \\
\Psi\left(x^{(1)}, x^{(2)}\right) & =0.0001 \times\left[x^{(1)}\left(1-x^{(1)}\right) x^{(2)}\left(1-x^{(2)}\right)\right]^{2} .
\end{array}
$$

Denoting again by $u$ the solution obtained thanks to (16)-(25), we define the exact kinetics energy by $\overline{\mathcal{E}}_{c}(t)=\frac{1}{2} \int_{\Omega} \bar{u}^{2}(t, x) \mathrm{d} x$ and the approximate one by $\mathcal{E}_{c}(t)=\frac{1}{2} \int_{\Omega} u^{2}(t, x) \mathrm{d} x$. The exact kinetics energy follows the equation

$$
\left.\overline{\mathcal{E}}_{c}(t)=\overline{\mathcal{E}}_{c}(0)-t\left(\int_{\Omega} \nabla u_{0}(x): \nabla u_{0}(x) \mathrm{d} x+\varepsilon(t)\right), \forall t \in\right] 0, T[,
$$

with $\varepsilon(t) \rightarrow 0$ as $t \rightarrow 0$ (this relation states that the infinitesimal decay of $\overline{\mathcal{E}}_{c}$ only comes from the viscous term). Let us check that this is approximately verified by the discrete solution, using a mesh with $20 \times 20$ control volumes and a time step equal to $\delta t=10^{-7}$ (hence $T$ corresponds to a hundred time steps) and letting $\nu=10^{-7}$. We have $\overline{\mathcal{E}}_{c}(0)=\frac{4}{1323} \times 10^{6} \simeq 3023.43$ and $\int_{\Omega} \nabla u_{0}(x): \nabla u_{0}(x) \mathrm{d} x=\frac{16}{49} \times 10^{6} \simeq 3.27 \times 10^{5}$, and the computation of the numerical solution gives $\mathcal{E}_{c}(0) \simeq 3023.74$ and $\mathcal{E}_{c}(T) \simeq 3020.44$; this shows a decrease rate $\left[\mathcal{E}_{c}(0)-\mathcal{E}_{c}(T)\right] / T$ equal to $3.3 \times 10^{5}$, very close to the theoretical value $3.27 \times 10^{5}$. This example shows that the mixed finite volume scheme does not introduce any significant artificial energy decay: the only decay comes from the diffusion term, and the discretization of the convective nonlinear term induces no additional diffusion phenomenon (as we will prove during the analysis of the scheme - see $(37))$.

\section{Mathematical study of the scheme for Stokes problem}

Here are the results we prove on the scheme for Stokes problem.

Theorem 4.1 [Existence of a unique solution to the scheme for Stokes problem] Assume that (2) and (3) hold. Let $\mathcal{D}$ be an admissible discretization of $\Omega$ in the sense of Definition 2.1 and let $\nu>0$. Then there exists a unique $\left(p,\left(u^{(i)}, \mathbf{v}^{(i)}, \widetilde{F}^{(i)}\right)_{i=1, \ldots, d}\right) \in H_{\mathcal{D}} \times$ $\left(H_{\mathcal{D}} \times H_{\mathcal{D}}^{d} \times \mathcal{F}_{\mathcal{D}}\right)^{d}$ solution to $(9)-(15)$.

Theorem 4.2 [Convergence of the scheme for Stokes problem] Assume that (2) and (3) hold. Let $\left(\mathcal{D}_{m}\right)_{m \geq 1}$ be a sequence of admissible discretizations of $\Omega$ in the sense of Definition 2.1, 
such that $\operatorname{size}\left(\mathcal{D}_{m}\right) \rightarrow 0$ as $m \rightarrow \infty$ and $\left(\operatorname{regul}\left(\mathcal{D}_{m}\right)\right)_{m \geq 1}$ is bounded. Let $\lambda>0$ and $\left.\alpha \in\right] 0,2[$, and define $\nu_{m}=\lambda \operatorname{size}\left(\mathcal{D}_{m}\right)^{\alpha}$. Let $\left(p_{m},\left(u_{m}^{(i)}, \mathbf{v}_{m}^{(i)}, \widetilde{F}_{m}^{(i)}\right)_{i=1, \ldots, d}\right)$ be the solution to (9)-(15) with $\mathcal{D}=\mathcal{D}_{m}$ and $\nu=\nu_{m}$. Let $\bar{u}$ be the unique solution to (4) and denote $u_{m}=\left(u_{m}^{(1)}, \ldots, u_{m}^{(d)}\right)$ and $\mathbf{v}_{m}=\left(\mathbf{v}_{m}^{(1)}, \ldots, \mathbf{v}_{m}^{(d)}\right)$.

Then, as $m \rightarrow \infty, u_{m} \rightarrow \bar{u}$ strongly in $L^{q}(\Omega)^{d}$ for all $q<\frac{2 d}{d-2}$ (and weakly in $L^{6}(\Omega)^{3}$ if $d=3$ ) and $\mathbf{v}_{m} \rightarrow \nabla \bar{u}$ strongly in $L^{2}(\Omega)^{d \times d}$.

\subsection{A priori estimates}

As it is usual in finite volume schemes, the proof of convergence relies on a priori estimates on the solution to the scheme.

Proposition 4.1 [A priori estimates on $\mathbf{v}$ and $\widetilde{F}$ ] Assume that (2) and (3) hold. Let $\mathcal{D}$ be an admissible discretization of $\Omega$ in the sense of Definition 2.1 and $\theta \geq \operatorname{regul}(\mathcal{D})$. Let $\nu_{0}>0$ and assume that $0 \leq \nu \leq \nu_{0}$. If $\left(p,\left(u^{(i)}, \mathbf{v}^{(i)}, \widetilde{F}^{(i)}\right)_{i=1, \ldots, d}\right) \in H_{\mathcal{D}} \times\left(H_{\mathcal{D}} \times H_{\mathcal{D}}^{d} \times \mathcal{F}_{\mathcal{D}}\right)^{d}$ is a solution to (9)-(15), then

$$
\sum_{i=1}^{d}\left\|\mathbf{v}^{(i)}\right\|_{L^{2}(\Omega)^{d}}^{2}+\sum_{i=1}^{d} \sum_{K \in \mathcal{M}} \sum_{\sigma \in \mathcal{E}_{K}} \nu \frac{\operatorname{diam}(K)}{\mathrm{m}(\sigma)}\left|\widetilde{F}_{K, \sigma}^{(i)}\right|^{2} \leq C_{1}\|f\|_{L^{2}(\Omega)^{d}}^{2},
$$

where $C_{1}$ only depends on $d, \Omega, \theta$ and $\nu_{0}$.

Proof of Proposition 4.1

Multiply (14) by $u_{K}^{(i)}$ and sum on $i$ and on the control volumes $K$. Using the conservation property (11), we can gather by edges to find

$$
\sum_{i=1}^{d} \sum_{\sigma^{K \mid L} \in \mathcal{E}_{\text {int }}} F_{K, \sigma}^{(i)}\left(u_{L}^{(i)}-u_{K}^{(i)}\right)+\sum_{i=1}^{d} \sum_{\sigma^{K \mid \partial} \in \mathcal{E}_{\text {ext }}} F_{K, \sigma}^{(i)}\left(-u_{K}^{(i)}\right)=\sum_{i=1}^{d} \int_{\Omega} f^{(i)}(x) u^{(i)}(x) \mathrm{d} x .
$$

Thanks to (9) and to the conservation of $F_{K, \sigma}^{(i)}$, we deduce, gathering by control volumes,

$$
\begin{gathered}
\sum_{i=1}^{d} \sum_{K \in \mathcal{M}} \mathbf{v}_{K}^{(i)} \cdot \sum_{\sigma \in \mathcal{E}_{K}} F_{K, \sigma}^{(i)}\left(\mathbf{x}_{\sigma}-\mathbf{x}_{K}\right)+\sum_{i=1}^{d} \sum_{K \in \mathcal{M}} \sum_{\sigma \in \mathcal{E}_{K}} \nu \frac{\operatorname{diam}(K)}{\mathrm{m}(\sigma)} F_{K, \sigma}^{(i)} \widetilde{F}_{K, \sigma}^{(i)} \\
=\sum_{i=1}^{d} \int_{\Omega} f^{(i)}(x) u^{(i)}(x) \mathrm{d} x .
\end{gathered}
$$

From (10), (12) and Lemma 6.1, we have $\sum_{\sigma \in \mathcal{E}_{K}} F_{K, \sigma}^{(i)}\left(\mathbf{x}_{\sigma}-\mathbf{x}_{K}\right)=\mathrm{m}(K) \mathbf{v}_{K}^{(i)}-\mathrm{m}(K) p_{K} \mathbf{e}^{(i)}$ and therefore, using (13),

$$
\begin{aligned}
& \sum_{i=1}^{d} \sum_{K \in \mathcal{M}} \mathbf{v}_{K}^{(i)} \cdot \sum_{\sigma \in \mathcal{E}_{K}} F_{K, \sigma}^{(i)}\left(\mathbf{x}_{\sigma}-\mathbf{x}_{K}\right) \\
& \quad=\sum_{i=1}^{d}\left\|\mathbf{v}^{(i)}\right\|_{L^{2}(\Omega)^{d}}^{2}-\sum_{K \in \mathcal{M}} \mathrm{m}(K) p_{K} \sum_{i=1}^{d} \mathbf{v}_{K}^{(i)} \cdot \mathbf{e}^{(i)} \\
& \quad=\sum_{i=1}^{d}\left\|\mathbf{v}^{(i)}\right\|_{L^{2}(\Omega)^{d}}^{2}+\sum_{K \in \mathcal{M}} \nu \operatorname{diam}(K) p_{K} \sum_{i=1}^{d} \sum_{\sigma \in \mathcal{E}_{K}} \widetilde{F}_{K, \sigma}^{(i)} \mathbf{e}^{(i)} \cdot \mathbf{n}_{K, \sigma}
\end{aligned}
$$


On the other hand, from (10),

$$
\begin{aligned}
& \sum_{i=1}^{d} \sum_{K \in \mathcal{M}} \sum_{\sigma \in \mathcal{E}_{K}} \nu \frac{\operatorname{diam}(K)}{\mathrm{m}(\sigma)} F_{K, \sigma}^{(i)} \widetilde{F}_{K, \sigma}^{(i)} \\
& \quad=\sum_{i=1}^{d} \sum_{K \in \mathcal{M}} \sum_{\sigma \in \mathcal{E}_{K}} \nu \frac{\operatorname{diam}(K)}{\mathrm{m}(\sigma)}\left|\widetilde{F}_{K, \sigma}^{(i)}\right|^{2}-\sum_{i=1}^{d} \sum_{K \in \mathcal{M}} \sum_{\sigma \in \mathcal{E}_{K}} \nu \operatorname{diam}(K) p_{K} \mathbf{e}^{(i)} \cdot \mathbf{n}_{K, \sigma} \widetilde{F}_{K, \sigma}^{(i)} .
\end{aligned}
$$

Adding together (28) and (29), we notice that the terms involving $\widetilde{F}_{K, \sigma}^{(i)}$ and $p_{K}$ disappear and, coming back to $(27)$, we obtain

$$
\sum_{i=1}^{d}\left\|\mathbf{v}^{(i)}\right\|_{L^{2}(\Omega)^{d}}^{2}+\sum_{i=1}^{d} \sum_{K \in \mathcal{M}} \sum_{\sigma \in \mathcal{E}_{K}} \nu \frac{\operatorname{diam}(K)}{\mathrm{m}(\sigma)}\left|\widetilde{F}_{K, \sigma}^{(i)}\right|^{2}=\sum_{i=1}^{d} \int_{\Omega} f^{(i)}(x) u^{(i)}(x) \mathrm{d} x .
$$

Using Young's inequality and Lemma 6.2, we have, for all $\varepsilon>0$,

$$
\begin{aligned}
\int_{\Omega} f^{(i)}(x) u^{(i)}(x) \mathrm{d} x & \leq \frac{1}{2 \varepsilon}\left\|f^{(i)}\right\|_{L^{2}(\Omega)}^{2}+\frac{\varepsilon}{2}\left\|u^{(i)}\right\|_{L^{2}(\Omega)}^{2} \\
& \leq \frac{1}{2 \varepsilon}\left\|f^{(i)}\right\|_{L^{2}(\Omega)}^{2}+\varepsilon C_{2}\left\|\mathbf{v}^{(i)}\right\|_{L^{2}(\Omega)^{d}}^{2}+\varepsilon C_{2} \sum_{K \in \mathcal{M}} \sum_{\sigma \in \mathcal{E}_{K}} \nu^{2} \frac{\operatorname{diam}(K)}{\mathrm{m}(\sigma)}\left|\widetilde{F}_{K, \sigma}^{(i)}\right|^{2} \\
& \leq \frac{1}{2 \varepsilon}\left\|f^{(i)}\right\|_{L^{2}(\Omega)}^{2}+\varepsilon C_{2}\left\|\mathbf{v}^{(i)}\right\|_{L^{2}(\Omega)^{d}}^{2}+\varepsilon C_{2} \nu_{0} \sum_{K \in \mathcal{M}} \sum_{\sigma \in \mathcal{E}_{K}} \nu \frac{\operatorname{diam}(K)}{\operatorname{m}(\sigma)}\left|\widetilde{F}_{K, \sigma}^{(i)}\right|^{2} .
\end{aligned}
$$

where $C_{2}$ only depends on $d, \Omega$ and $\theta$. The proof is concluded by taking $\varepsilon=\inf \left(\frac{1}{2 C_{2}}, \frac{1}{2 \nu_{0} C_{2}}\right)$ and injecting the result in (30).

\subsection{Proof of the theorems}

Using the preceding estimates, we can now prove the existence of a unique solution to (9) - (15).

Proof OF THEOREM 4.1

As explained in Remark 2.2, (9) - (15) can in fact be considered as a square system (as many equations as there are unknowns). Since this system is linear, Proposition 4.1 shows that, if the terms $\int_{K} f^{(i)} \mathrm{d} x$ in the right-hand side of $(9)-(15)$ are null then $\left(\mathbf{v}^{(i)}, \widetilde{F}^{(i)}\right)_{i=1, \ldots, d}$ are null, which in turn imply (thanks to (9)) that $\left(u^{(i)}\right)_{i=1, \ldots, d}$ is null. From (10) and (11), we deduce $p_{K}=p_{L}$ for all neighboring control volumes $K$ and $L$; since $\Omega$ is connected this means that $p$ is constant and, by (15), that it is null. Hence, the square system (9)-(15) is well-posed and has a unique solution.

Let us now show that the approximate solution converges to the weak solution of (1).

Proof of Theorem 4.2

To simply the notations, we drop the index $m$ in $\mathcal{D}_{m}, p_{m}, u_{m}^{(i)}, \mathbf{v}_{m}^{(i)}$ and $\widetilde{F}_{m}^{(i)}$. As it is usual, since the solution to (4) is unique, it is enough to prove the convergence of a subsequence of $(u, \mathbf{v})$ toward the solution of this problem. 
Proposition 4.1 gives estimates on $\left(\left(\mathbf{v}^{(i)}, \widetilde{F}^{(i)}\right)_{i=1, \ldots, d}\right)$ which are uniform with respect to $\mathcal{D}$, since $\operatorname{regul}(\mathcal{D})$ and $\nu=\lambda \operatorname{size}(\mathcal{D})^{\alpha}$ are bounded. We can therefore write, for all $i=1, \ldots, d$,

$$
\sum_{K \in \mathcal{M}} \sum_{\sigma \in \mathcal{E}_{K}} \nu^{2} \frac{\operatorname{diam}(K)}{\mathrm{m}(\sigma)}\left|\widetilde{F}_{K, \sigma}^{(i)}\right|^{2} \leq C_{3} \nu=C_{3} \lambda \operatorname{size}(\mathcal{D})^{\alpha}
$$

with $C_{3}$ not depending on $\mathcal{D}$. This last quantity tends to 0 as $\operatorname{size}(\mathcal{D}) \rightarrow 0$ and by Lemma 6.4 we deduce that there exists $\bar{u}=\left(\bar{u}^{(1)}, \ldots, \bar{u}^{(d)}\right) \in H_{0}^{1}(\Omega)^{d}$ such that, up to a subsequence as $\operatorname{size}(\mathcal{D}) \rightarrow 0, u \rightarrow \bar{u}$ strongly in $L^{q}(\Omega)^{d}$ for all $q<\frac{2 d}{d-2}$ (and weakly in $L^{6}(\Omega)^{d}$ if $d=3$ ) and $\mathbf{v} \rightarrow \nabla \bar{u}$ weakly in $L^{2}(\Omega)^{d \times d}$.

Step 1: $\bar{u}$ belongs to $E(\Omega)$.

Let $\Gamma: \Omega \rightarrow \mathbb{R}$ be the piecewise function equal to $\frac{\nu \operatorname{diam}(K)}{\mathrm{m}(K)} \sum_{i=1}^{d} \sum_{\sigma \in \mathcal{E}_{K}} \widetilde{F}_{K, \sigma}^{(i)} \mathbf{e}^{(i)} \cdot \mathbf{n}_{K, \sigma}$ on $K \in \mathcal{M}$. From Cauchy-Schwarz inequality, we have

$$
\begin{aligned}
\|\Gamma\|_{L^{1}(\Omega)} & =\sum_{K \in \mathcal{M}}\left|\sum_{i=1}^{d} \sum_{\sigma \in \mathcal{E}_{K}} \nu \operatorname{diam}(K) \widetilde{F}_{K, \sigma}^{(i)} \mathbf{e}^{(i)} \cdot \mathbf{n}_{K, \sigma}\right| \\
& \leq\left(\sum_{i=1}^{d} \sum_{K \in \mathcal{M}} \sum_{\sigma \in \mathcal{E}_{K}} \nu^{2} \frac{\operatorname{diam}(K)}{\mathrm{m}(\sigma)}\left|\widetilde{F}_{K, \sigma}^{(i)}\right|^{2}\right)^{1 / 2}\left(\sum_{i=1}^{d} \sum_{K \in \mathcal{M}} \sum_{\sigma \in \mathcal{E}_{K}} \operatorname{diam}(K) \mathrm{m}(\sigma)\right)^{1 / 2}
\end{aligned}
$$

Hence, using (8) and (31), we have $\Gamma \rightarrow 0$ in $L^{1}(\Omega)$ as $\operatorname{size}(\mathcal{D}) \rightarrow 0$. Since $\sum_{i=1}^{d} \mathbf{v}^{(i)} \cdot \mathbf{e}^{(i)}+\Gamma=0$ on $\Omega$ (this is (13) divided by $\mathrm{m}(K)$ ), we deduce from the weak convergence of $\mathbf{v}^{(i)}$ to $\nabla \bar{u}^{(i)}$ that $\operatorname{div}(\bar{u})=\sum_{i=1}^{d} \nabla \bar{u}^{(i)} \cdot \mathbf{e}^{(i)}=0$ and thus that $\bar{u} \in E(\Omega)$.

Step 2: $\bar{u}$ satisfies (4).

By the density of $\left\{\varphi \in C_{c}^{\infty}(\Omega)^{d}, \operatorname{div}(\varphi)=0\right\}$ in $E(\Omega)$ (see [17]), it is sufficient to prove (4) for $\varphi$ regular with compact support. Let $\varphi$ be such a function; we multiply $(14)$ by $\varphi^{(i)}\left(\mathbf{x}_{K}\right)$ and we sum on $K$ and $i$ :

$$
-\sum_{i=1}^{d} \sum_{K \in \mathcal{M}} \sum_{\sigma \in \mathcal{E}_{K}} F_{K, \sigma}^{(i)} \varphi^{(i)}\left(\mathbf{x}_{K}\right)=\sum_{i=1}^{d} \sum_{K \in \mathcal{M}} \int_{K} \varphi^{(i)}\left(\mathbf{x}_{K}\right) f^{(i)}(x) \mathrm{d} x .
$$

Let $\varphi_{\sigma}^{(i)}=\frac{1}{\mathrm{~m}(\sigma)} \int_{\sigma} \varphi^{(i)}(x) \mathrm{d} \gamma(x)$. By $(11)$ and since $\varphi_{\sigma}^{(i)}=0$ for $\sigma \in \mathcal{E}_{\text {ext }}$, we have

$$
\sum_{K \in \mathcal{M}} \sum_{\sigma \in \mathcal{E}_{K}} F_{K, \sigma}^{(i)} \varphi_{\sigma}^{(i)}=\sum_{\sigma^{K \mid L} \in \mathcal{E}_{\mathrm{int}}}\left(F_{K, \sigma}^{(i)}+F_{L, \sigma}^{(i)}\right) \varphi_{\sigma}^{(i)}=0 .
$$

Hence, $(32)$ can be written, with $\varphi_{\mathcal{D}}^{(i)}$ equal to $\varphi^{(i)}\left(\mathbf{x}_{K}\right)$ on each mesh $K$,

$$
\sum_{i=1}^{d} \sum_{K \in \mathcal{M}} \sum_{\sigma \in \mathcal{E}_{K}} F_{K, \sigma}^{(i)}\left(\varphi_{\sigma}^{(i)}-\varphi^{(i)}\left(\mathbf{x}_{K}\right)\right)=\sum_{i=1}^{d} \int_{\Omega} \varphi_{\mathcal{D}}^{(i)}(x) f^{(i)}(x) \mathrm{d} x .
$$

We have, since $\sum_{\sigma \in \mathcal{E}_{K}} \mathrm{~m}(\sigma) \mathbf{n}_{K, \sigma}=0$ and $\operatorname{div}(\varphi)=0$,

$$
\begin{aligned}
& \sum_{i=1}^{d} \sum_{K \in \mathcal{M}} p_{K} \sum_{\sigma \in \mathcal{E}_{K}} \mathrm{~m}(\sigma) \mathbf{e}^{(i)} \cdot \mathbf{n}_{K, \sigma}\left(\varphi_{\sigma}^{(i)}-\varphi^{(i)}\left(\mathbf{x}_{K}\right)\right) \\
& \quad=\sum_{K \in \mathcal{M}} p_{K} \sum_{\sigma \in \mathcal{E}_{K}} \int_{\sigma} \sum_{i=1}^{d} \varphi^{(i)}(x) \mathbf{e}^{(i)} \cdot \mathbf{n}_{K, \sigma} \mathrm{d} \gamma(x)=\sum_{K \in \mathcal{M}} p_{K} \int_{K} \operatorname{div}(\varphi)(x) \mathrm{d} x=0
\end{aligned}
$$


and therefore, from (33) and (10), we deduce

$$
\sum_{i=1}^{d} \sum_{K \in \mathcal{M}} \sum_{\sigma \in \mathcal{E}_{K}} \widetilde{F}_{K, \sigma}^{(i)}\left(\varphi_{\sigma}^{(i)}-\varphi^{(i)}\left(\mathbf{x}_{K}\right)\right)=\sum_{i=1}^{d} \int_{\Omega} \varphi_{\mathcal{D}}^{(i)}(x) f^{(i)}(x) \mathrm{d} x
$$

Since $\mathbf{x}_{\sigma}$ is the gravity center of $\sigma$ and $\varphi_{\sigma}^{(i)}$ is the mean value on $\sigma$ of the regular function $\varphi^{(i)}$, we have $\varphi_{\sigma}^{(i)}-\varphi^{(i)}\left(\mathbf{x}_{K}\right)=\frac{1}{\mathrm{~m}(K)} \int_{K} \nabla \varphi^{(i)}(x) \mathrm{d} x \cdot\left(\mathbf{x}_{\sigma}-\mathbf{x}_{K}\right)+R_{K, \sigma}^{(i)}$ with $\left|R_{K, \sigma}^{(i)}\right| \leq C_{\varphi} \operatorname{diam}(K)^{2}$ (where $C_{\varphi}$ only depends on $\varphi$ ). From (34), we deduce

$$
\sum_{i=1}^{d} \sum_{K \in \mathcal{M}} \frac{1}{\mathrm{~m}(K)} \int_{K} \nabla \varphi^{(i)}(x) \mathrm{d} x \cdot \sum_{\sigma \in \mathcal{E}_{K}} \widetilde{F}_{K, \sigma}^{(i)}\left(\mathbf{x}_{\sigma}-\mathbf{x}_{K}\right)=\sum_{i=1}^{d} \int_{\Omega} f^{(i)}(x) \varphi_{\mathcal{D}}^{(i)}(x) \mathrm{d} x+T_{1},
$$

where $\left|T_{1}\right| \leq C_{\varphi} \sum_{i=1}^{d} \sum_{K \in \mathcal{M}} \sum_{\sigma \in \mathcal{E}_{K}}\left|\widetilde{F}_{K, \sigma}^{(i)}\right| \operatorname{diam}(K)^{2}$. Using then (12), this gives

$$
\sum_{i=1}^{d} \int_{\Omega} \mathbf{v}^{(i)}(x) \cdot \nabla \varphi^{(i)}(x) \mathrm{d} x=\sum_{i=1}^{d} \int_{\Omega} f^{(i)}(x) \varphi_{\mathcal{D}}^{(i)}(x) \mathrm{d} x+T_{1} .
$$

By weak convergence of $\mathbf{v}$ to $\nabla \bar{u}$ and regularity of $\varphi$, the first two terms of this equality respectively converge to $\int_{\Omega} \nabla \bar{u}(x): \nabla \varphi(x) \mathrm{d} x$ and $\int_{\Omega} f(x) \cdot \varphi(x) \mathrm{d} x$ as $\operatorname{size}(\mathcal{D}) \rightarrow 0$. Hence, it remains to prove that $T_{1} \rightarrow 0$ to conclude the proof that $\bar{u}$ satisfies (4).

The convergence of $T_{1}$ is quite easy to establish thanks to Proposition 4.1. Indeed, from the estimates in this proposition and using (8), we have

$$
\begin{aligned}
\left|T_{1}\right| & \leq C_{4}\left(\sum_{i=1}^{d} \sum_{K \in \mathcal{M}} \sum_{\sigma \in \mathcal{E}_{K}} \operatorname{diam}(K)^{4} \frac{\mathrm{m}(\sigma)}{\nu \operatorname{diam}(K)}\right)^{1 / 2} \\
& \leq C_{4}\left(\sum_{i=1}^{d} \sum_{K \in \mathcal{M}} \sum_{\sigma \in \mathcal{E}_{K}} \frac{\operatorname{size}(\mathcal{D})^{2}}{\lambda \operatorname{size}(\mathcal{D})^{\alpha}} \mathrm{m}(\sigma) \operatorname{diam}(K)\right)^{1 / 2} \leq C_{5} \operatorname{size}(\mathcal{D})^{\frac{2-\alpha}{2}}
\end{aligned}
$$

where $C_{4}$ and $C_{5}$ do not depend on $\mathcal{D}$. Since $\alpha<2$, this last term tends to 0 as $\operatorname{size}(\mathcal{D}) \rightarrow 0$, which concludes the proof that $\bar{u}$ is the weak solution to the Stokes equation.

Step 3: it remains to prove that the convergence of $\mathbf{v}$ to $\nabla \bar{u}$ is strong.

In order to do so, we recall (30), which implies

$$
\sum_{i=1}^{d}\left\|\mathbf{v}^{(i)}\right\|_{L^{2}(\Omega)^{d}}^{2} \leq \sum_{i=1}^{d} \int_{\Omega} f^{(i)}(x) u^{(i)}(x) \mathrm{d} x .
$$

By convergence of $u$ to $\bar{u}$, and since $\bar{u}$ is a solution to (4), we deduce

$$
\limsup _{\operatorname{size}(\mathcal{D}) \rightarrow 0} \sum_{i=1}^{d}\left\|\mathbf{v}^{(i)}\right\|_{L^{2}(\Omega)^{d}}^{2} \leq \sum_{i=1}^{d} \int_{\Omega} f^{(i)}(x) \bar{u}^{(i)}(x) \mathrm{d} x=\sum_{i=1}^{d} \int_{\Omega}\left|\nabla \bar{u}^{(i)}(x)\right|^{2} \mathrm{~d} x .
$$

On the other hand, since $\mathbf{v}^{(i)} \rightarrow \nabla \bar{u}^{(i)}$ weakly in $L^{2}(\Omega)^{d}$,

$$
\sum_{i=1}^{d} \int_{\Omega}\left|\nabla \bar{u}^{(i)}(x)\right|^{2} \mathrm{~d} x=\sum_{i=1}^{d}\left\|\nabla \bar{u}^{(i)}\right\|_{L^{2}(\Omega)^{d}}^{2} \leq \liminf _{\operatorname{size}(\mathcal{D}) \rightarrow 0} \sum_{i=1}^{d}\left\|\mathbf{v}^{(i)}\right\|_{L^{2}(\Omega)^{d}}^{2}
$$


and therefore $\sum_{i=1}^{d}\left\|\mathbf{v}^{(i)}\right\|_{L^{2}(\Omega)^{d}}^{2} \rightarrow \sum_{i=1}^{d}\left\|\nabla \bar{u}^{(i)}\right\|_{L^{2}(\Omega)^{d}}^{2}$ as size $(\mathcal{D}) \rightarrow 0$. Since the expression $\left(\sum_{i=1}^{d}\|\cdot\|_{L^{2}(\Omega) d}^{2}\right)^{1 / 2}$ is a Hilbert norm on $L^{2}(\Omega)^{d \times d}$, the weak convergence of $\mathbf{v}$ to $\nabla \bar{u}$ and the convergence of the norm of $\mathbf{v}$ toward the norm of $\nabla \bar{u}$ imply the strong convergence of $\mathbf{v}$ in $L^{2}(\Omega)^{d \times d}$.

\section{Mathematical study of the scheme for Navier-Stokes problem}

Here are the two results we prove on the scheme (16)-(25).

Theorem 5.1 [Existence of a solution to the scheme for Navier-Stokes problem] Let $T>0$ and assume that (2) and (6) hold. Let $\delta t>0$ such that $N_{\delta t}=T / \delta t$ is an integer. Let $\mathcal{D}$ be an admissible discretization of $\Omega$ in the sense of Definition 2.1 and let $\nu>0$. Then there exists at least one $\left(p,\left(u^{(i)}, \mathbf{v}^{(i)}, \widetilde{F}^{(i)}\right)_{i=1, \ldots, d}\right) \in H_{\mathcal{D}, \delta t} \times\left(H_{\mathcal{D}, \delta t} \times H_{\mathcal{D}, \delta t}^{d} \times \mathcal{F}_{\mathcal{D}, \delta t}\right)^{d}$ solution to (16)—(25).

Theorem 5.2 [Convergence of the scheme for Navier-Stokes problem] Let $T>0$ and assume that (2) and (6) hold. Let $\delta_{m} \rightarrow 0$ such that, for all $m \geq 1, N_{\delta t_{m}}=T / \delta t_{m}$ is an integer. Let $\left(\mathcal{D}_{m}\right)_{m \geq 1}$ be a sequence of admissible discretizations of $\Omega$ in the sense of Definition 2.1, such that $\operatorname{size}\left(\mathcal{D}_{m}\right) \rightarrow 0$ as $m \rightarrow \infty$ and $\left(\operatorname{regul}\left(\mathcal{D}_{m}\right)\right)_{m \geq 1}$ is bounded. Let $\lambda>0$ and $\left.\alpha \in\right] 0,2[$, and define $\nu_{m}=\lambda \operatorname{size}\left(\mathcal{D}_{m}\right)^{\alpha}$. Let $\left(p_{m},\left(u_{m}^{(i)}, \mathbf{v}_{m}^{(i)}, \widetilde{F}_{m}^{(i)}\right)_{i=1, \ldots, d}\right)$ be the solution to (16) - (25) with $\delta t=\delta t_{m}, \mathcal{D}=\mathcal{D}_{m}$ and $\nu=\nu_{m}$. Let $u_{m}=\left(u_{m}^{(1)}, \ldots, u_{m}^{(d)}\right)$ and $\mathbf{v}_{m}=\left(\mathbf{v}_{m}^{(1)}, \ldots, \mathbf{v}_{m}^{(d)}\right)$.

Then there exists a weak solution $\bar{u}$ to (5) such that, up to a subsequence as $m \rightarrow \infty, u_{m} \rightarrow \bar{u}$ strongly in $L^{2}(] 0, T[\times \Omega)^{d}$ and $\mathbf{v}_{m} \rightarrow \nabla \bar{u}$ weakly in $L^{2}(] 0, T[\times \Omega)^{d \times d}$.

Remark 5.1 In dimension $d=2$, the solution $\bar{u}$ is unique and has enough regularity to be used as a test function in (7) (see [17]). Hence, in this case, the whole sequence of approximate solutions converge toward the weak solution and we can mimic the method used in [6], [4] or the proof of Theorem 4.2 to prove that the convergence of $\mathbf{v}$ is in fact strong in $L^{2}(] 0, T[\times \Omega)^{d \times d}$.

\subsection{A priori estimates and existence of an approximate solution}

We begin with a priori estimates, similar to the ones obtained for the scheme on the Stokes problem.

Proposition 5.1 Let $T>0$ and take $\delta t>0$ such that $N_{\delta t}=T / \delta t$ is a positive integer. Assume that (2) and (6) hold. Let $\mathcal{D}$ be an admissible discretization of $\Omega$ in the sense of Definition 2.1 and $\theta \geq \operatorname{regul}(\mathcal{D})$. Let $0<\nu \leq \nu_{0}$. If $\left(p,\left(u^{(i)}, \mathbf{v}^{(i)}, \widetilde{F}^{(i)}\right)_{i=1, \ldots, d}\right) \in H_{\mathcal{D}, \delta t} \times\left(H_{\mathcal{D}, \delta t} \times H_{\mathcal{D}, \delta t}^{d} \times \mathcal{F}_{\mathcal{D}, \delta t}\right)^{d}$ is a solution to (16) - (25) then

$$
\sum_{i=1}^{d}\left\|u^{(i)}\right\|_{L^{\infty}\left(0, T ; L^{2}(\Omega)\right)}^{2}+\sum_{i=1}^{d}\left\|\mathbf{v}^{(i)}\right\|_{L^{2}(] 0, T[\times \Omega)^{d}}^{2} \leq C_{6}\left(\|f\|_{L^{2}(] 0, T[\times \Omega)^{d}}^{2}+\left\|u_{0}\right\|_{L^{2}(\Omega)^{d}}^{2}\right)
$$

and

$$
\sum_{i=1}^{d} \sum_{n=0}^{N_{\delta t}-1} \delta t \sum_{K \in \mathcal{M}} \sum_{\sigma \in \mathcal{E}_{K}} \nu \frac{\operatorname{diam}(K)}{\mathrm{m}(\sigma)}\left|\left(\widetilde{F}^{(i)}\right)_{K, \sigma}^{n+1 / 2}\right|^{2} \leq C_{6}\left(\|f\|_{L^{2}(] 0, T[\times \Omega)^{d}}^{2}+\left\|u_{0}\right\|_{L^{2}(\Omega)^{d}}^{2}\right)
$$

where $C_{6}$ only depends on $d, \Omega, T, \theta$ and $\nu_{0}$. 
Proof of Proposition 5.1

Multiply $(21)$ by $\delta t\left(u^{(i)}\right)_{K}^{n+1 / 2}=\delta t \frac{\left(u^{(i)}\right)_{K}^{n+1}+\left(u^{(i)}\right)_{K}^{n}}{2}$, sum on $K \in \mathcal{M}, i=1, \ldots, d$ and $n=$ $0, \ldots, N-1$ (with $1 \leq N \leq N_{\delta t}$ ). This gives $T_{2}+T_{3}+T_{4}=T_{5}$ with

$$
\begin{aligned}
T_{2} & =\frac{1}{2} \sum_{i=1}^{d} \sum_{n=0}^{N-1} \sum_{K \in \mathcal{M}} \mathrm{m}(K)\left(\left[\left(u^{(i)}\right)_{K}^{n+1}\right]^{2}-\left[\left(u^{(i)}\right)_{K}^{n}\right]^{2}\right) \\
T_{3} & =\sum_{i=1}^{d} \sum_{n=0}^{N-1} \delta t \sum_{K \in \mathcal{M}} \sum_{\sigma \in \mathcal{E}_{K}} \mathrm{~m}(\sigma) \vec{u}_{\sigma}^{n+1 / 2} \cdot \mathbf{n}_{K, \sigma}\left(\frac{\left(u^{(i)}\right)_{K}^{n+1 / 2}+\left(u^{(i)}\right)_{L}^{n+1 / 2}}{2}\right)\left(u^{(i)}\right)_{K}^{n+1 / 2} \\
T_{4} & =-\sum_{n=0}^{N-1} \delta t \sum_{i=1}^{d} \sum_{K \in \mathcal{M}} \sum_{\sigma \in \mathcal{E}_{K}}\left(F^{(i)}\right)_{K, \sigma}^{n+1 / 2}\left(u^{(i)}\right)_{K}^{n+1 / 2} \\
T_{5} & =\int_{0}^{N \delta t} \int_{\Omega} f^{(i)}(t, x) u^{(i)}(t, x) \mathrm{d} t \mathrm{~d} x
\end{aligned}
$$

(in $T_{3}$, recall that $L$ denotes the neighboring control volume of $K$ on the other side of $\sigma$, if $\sigma \in \mathcal{E}_{K \text {,int }}$, or that $\left(u^{(i)}\right)_{L}^{n+1 / 2}=0$ if $\left.\sigma \in \mathcal{E}_{K \text {,ext }}\right)$. We clearly have, denoting $\left(u^{(i)}\right)^{N} \in H_{\mathcal{D}}$ the function equal to $\left(u^{(i)}\right)_{K}^{N}$ on $K \in \mathcal{M}$,

$$
T_{2}=\frac{1}{2} \sum_{i=1}^{d}\left\|\left(u^{(i)}\right)^{N}\right\|_{L^{2}(\Omega)}^{2}-\left\|\left(u^{(i)}\right)^{0}\right\|_{L^{2}(\Omega)}^{2} .
$$

Gathering by edges and denoting $\sigma=\sigma^{K \mid L}$ if $\sigma \in \mathcal{E}_{\text {int }}$, or $\left(u^{(i)}\right)_{L}^{n+1 / 2}=0$ if $\sigma=\sigma^{K \mid \partial} \in \mathcal{E}_{\text {ext }}$, we can write

$$
\begin{aligned}
T_{3} & =\sum_{i=1}^{d} \sum_{n=0}^{N-1} \delta t \sum_{\sigma \in \mathcal{E}} \mathrm{m}(\sigma) \vec{u}_{\sigma}^{n+1 / 2} \cdot \mathbf{n}_{K, \sigma}\left(\frac{\left(u^{(i)}\right)_{K}^{n+1 / 2}+\left(u^{(i)}\right)_{L}^{n+1 / 2}}{2}\right)\left(\left(u^{(i)}\right)_{K}^{n+1 / 2}-\left(u^{(i)}\right)_{L}^{n+1 / 2}\right) \\
& =\frac{1}{2} \sum_{i=1}^{d} \sum_{n=0}^{N-1} \delta t \sum_{\sigma \in \mathcal{E}} \mathrm{m}(\sigma) \vec{u}_{\sigma}^{n+1 / 2} \cdot \mathbf{n}_{K, \sigma}\left(\left[\left(u^{(i)}\right)_{K}^{n+1 / 2}\right]^{2}-\left[\left(u^{(i)}\right)_{L}^{n+1 / 2}\right]^{2}\right) .
\end{aligned}
$$

We now gather back by control volumes and we find, thanks to (26),

$$
T_{3}=\frac{1}{2} \sum_{i=1}^{d} \sum_{n=0}^{N-1} \delta t \sum_{K \in \mathcal{M}}\left[\left(u^{(i)}\right)_{K}^{n+1 / 2}\right]^{2} \sum_{\sigma \in \mathcal{E}_{K}} \mathrm{~m}(\sigma) \vec{u}_{\sigma}^{n+1 / 2} \cdot \mathbf{n}_{K, \sigma}=0 .
$$

The term $T_{4}$ is handled exactly as in the Stokes equation (see the proof of Proposition 4.1) and gives the transient equivalent of the left-hand side (30)

$$
\begin{aligned}
T_{4} & =\sum_{n=0}^{N-1} \delta t \sum_{i=1}^{d}\left\|\left(\mathbf{v}^{(i)}\right)^{n+1 / 2}\right\|_{L^{2}(\Omega)^{d}}^{2}+\sum_{n=0}^{N-1} \delta t \sum_{i=1}^{d} \sum_{K \in \mathcal{M}} \sum_{\sigma \in \mathcal{E}_{K}} \nu \frac{\operatorname{diam}(K)}{\mathrm{m}(\sigma)}\left|\left(\widetilde{F}^{(i)}\right)_{K, \sigma}^{n+1 / 2}\right|^{2} \\
& =\sum_{i=1}^{d}\left\|\mathbf{v}^{(i)}\right\|_{L^{2}(] 0, N \delta t[\times \Omega)^{d}}^{2}+\sum_{n=0}^{N-1} \delta t \sum_{i=1}^{d} \sum_{K \in \mathcal{M}} \sum_{\sigma \in \mathcal{E}_{K}} \nu \frac{\operatorname{diam}(K)}{\mathrm{m}(\sigma)}\left|\left(\widetilde{F}^{(i)}\right)_{K, \sigma}^{n+1 / 2}\right|^{2} .
\end{aligned}
$$


We also have the following bound, independent on $N$ :

$$
T_{5} \leq \sum_{i=1}^{d}\left\|f^{(i)}\right\|_{L^{2}(] 0, T[\times \Omega)}\left\|u^{(i)}\right\|_{L^{2}(] 0, T[\times \Omega)} .
$$

We now gather (36), (37), (38) and (39) in $T_{2}+T_{3}+T_{4}=T_{5}$; since this relation is valid for any $N=1, \ldots, N_{\delta t}$ and since $\left\|\left(u^{(i)}\right)^{0}\right\|_{L^{2}(\Omega)} \leq\left\|u_{0}^{(i)}\right\|_{L^{2}(\Omega)}$ (see (24)), we deduce that

$$
\begin{aligned}
& \frac{1}{2} \sup _{N=0, \ldots, N_{\delta t}}\left(\sum_{i=1}^{d}\left\|\left(u^{(i)}\right)^{N}\right\|_{L^{2}(\Omega)}^{2}\right)+\sum_{i=1}^{d}\left\|\mathbf{v}^{(i)}\right\|_{L^{2}(] 0, T[\times \Omega)^{d}}^{2} \\
& +\sum_{n=0}^{N_{\delta t}-1} \delta t \sum_{i=1}^{d} \sum_{K \in \mathcal{M}} \sum_{\sigma \in \mathcal{E}_{K}} \nu \frac{\operatorname{diam}(K)}{\operatorname{m}(\sigma)}\left|\left(\widetilde{F}^{(i)}\right)_{K, \sigma}^{n+1 / 2}\right|^{2} \\
& \leq \frac{1}{2}\left\|u_{0}\right\|_{L^{2}(\Omega)^{d}}^{2}+\sum_{i=1}^{d}\left\|f^{(i)}\right\|_{L^{2}(] 0, T[\times \Omega)}\left\|u^{(i)}\right\|_{L^{2}(] 0, T[\times \Omega)} .
\end{aligned}
$$

For all $t \in] 0, T\left[, u^{(i)}(t, \cdot)\right.$ is equal, for some $n=0, \ldots, N_{\delta t}-1$, to $\left(u^{(i)}\right)^{n+1 / 2}=\frac{\left(u^{(i)}\right)^{n+1}+\left(u^{(i)}\right)^{n}}{2}$. Hence, $\left\|u^{(i)}\right\|_{L^{\infty}\left(0, T ; L^{2}(\Omega)\right)}^{2} \leq \sup _{N=0, \ldots, N_{\delta t}}\left\|\left(u^{(i)}\right)^{N}\right\|_{L^{2}(\Omega)}^{2}$ and Young's inequality (associated with the fact that $\left.\left\|u^{(i)}\right\|_{L^{2}(] 0, T[\times \Omega)}^{2} \leq T\left\|u^{(i)}\right\|_{L^{\infty}\left(0, T ; L^{2}(\Omega)\right)}^{2}\right)$ concludes the proof.

We can now prove the existence of at least one solution to the scheme for Navier-Stokes problem.

Proof OF THEOREM 5.1

Notice first that the a priori estimates of Proposition 5.1 still hold (with exactly the same $C_{6}$ ) if we multiply the second term of (21) (the only non-linear term of the scheme) by some $\beta \in[0,1]$. Moreover, from the estimates on $\widetilde{F}$ and (17) and (18), we deduce some estimates

on $p_{K}^{n+1 / 2}-p_{L}^{n+1 / 2}$ for neighboring control volumes $K$ and $L$; therefore, using (25), we obtain estimates on $p$ not depending on $\beta$ (these rough estimates however strongly depend on the mesh and the time step).

By the same reasoning as in Remark 2.2, we can consider that the non-linear system (16) - (25) is in fact square. The properties of the topological degree (see [5]) then imply that the degree of the function defining this system is equal to the degree of the same function without the non-linear term in (21). The resulting system is square and linear and the preceding estimates, which imply that any solution to this system is bounded, show that it is invertible. Hence, the linear function defining this system has a non-null topological degree, and so does the function defining (16) - (25). This shows that there exists at least one solution to the scheme.

\subsection{Translations estimates}

In order to pass to the limit in the nonlinear term of the equation, we need to obtain enough compactness on the approximate solution $u^{(i)}$, which demands some estimates on its translations in time (the translation in space being estimated thanks to Lemma 6.3). To prove those estimates, we introduce, for $\mathcal{D}$ an admissible discretization of $\Omega$ and $\nu>0$, the space $L_{\mathcal{D}, \nu}$ of the functions $\widehat{u}=\left(\widehat{u}^{(1)}, \ldots, \widehat{u}^{(d)}\right) \in H_{\mathcal{D}}^{d}$ for which there exists $(\widehat{\mathbf{v}}, \widehat{F})=\left(\widehat{\mathbf{v}}^{(i)}, \widehat{F}^{(i)}\right)_{i=1, \ldots, d} \in\left(H_{\mathcal{D}}^{d} \times \mathcal{F}_{\mathcal{D}}\right)^{d}$ such 
that $(\widehat{u}, \widehat{\mathbf{v}}, \widehat{F})$ satisfies $(9)$ and $(13)$. We call such $(\widehat{\mathbf{v}}, \widehat{F})$ "compatible" with $\widehat{u}$ and we endow $L_{\mathcal{D}, \nu}$ with the norm

$$
\begin{array}{r}
\|\widehat{u}\|_{L_{\mathcal{D}, \nu}}^{2}=\inf \left\{\sum_{i=1}^{d}\left\|\widehat{\mathbf{v}}^{(i)}\right\|_{L^{2}(\Omega)^{d}}^{2}+\sum_{i=1}^{d} \sum_{K \in \mathcal{M}} \sum_{\sigma \in \mathcal{E}_{K}} \nu \frac{\operatorname{diam}(K)}{\mathrm{m}(\sigma)}\left|\widehat{F}_{K, \sigma}^{(i)}\right|^{2} ;\right. \\
(\widehat{\mathbf{v}}, \widehat{F}) \text { is compatible with } \widehat{u}\}
\end{array}
$$

(notice that this infimum is in fact a minimum). Defining, for $\widehat{v} \in H_{\mathcal{D}}^{d}$, the norm

$$
\|\widehat{v}\|_{L_{\mathcal{D}, \nu}^{*}}=\sup \left\{\sum_{i=1}^{d} \sum_{K \in \mathcal{M}} \mathrm{m}(K) \widehat{v}_{K}^{(i)} \widehat{u}_{K}^{(i)} ; \widehat{u} \in L_{\mathcal{D}, \nu},\|\widehat{u}\|_{L_{\mathcal{D}, \nu}}=1\right\},
$$

we notice that, if $\widehat{u} \in L_{\mathcal{D}, \nu}$,

$$
\|\widehat{u}\|_{L^{2}(\Omega)^{d}}^{2} \leq\|\widehat{u}\|_{L_{\mathcal{D}, \nu}}\|\widehat{u}\|_{L_{\mathcal{D}, \nu}^{*}}
$$

These tools will allow us to prove the following estimate.

Proposition 5.2 Let $T>0$ and take $\delta t>0$ such that $N_{\delta t}=T / \delta t$ is a positive integer. Assume that (2) and (6) hold. Let $\mathcal{D}$ be an admissible discretization of $\Omega$ in the sense of Definition 2.1 and $\theta \geq \operatorname{regul}(\mathcal{D})$. Let $0<\nu \leq \nu_{0}$. If $\left(p,\left(u^{(i)}, \mathbf{v}^{(i)}, \widetilde{F}^{(i)}\right)_{i=1, \ldots, d}\right) \in H_{\mathcal{D}, \delta t} \times\left(H_{\mathcal{D}, \delta t} \times H_{\mathcal{D}, \delta t}^{d} \times \mathcal{F}_{\mathcal{D}, \delta t}\right)^{d}$ is a solution to (16) - (25) then, for all $\tau \in] 0, T[$,

$$
\int_{0}^{T-\tau}\|u(t+\tau, \cdot)-u(t, \cdot)\|_{L^{2}(\Omega)} \mathrm{d} t \leq C_{7} \sqrt{\tau}
$$

where $C_{7}$ only depends on $d, \Omega, T, \theta$ and $\nu_{0}$.

Proof of Proposition 5.2

In this proof, $C_{i}$ denote constants which only depend on $d, \Omega, T, \theta$ and $\nu_{0}$.

Step 1: we estimate the $L_{\mathcal{D}, \nu}^{*}$ norm of $\left(u^{(i)}\right)^{n+1}-\left(u^{(i)}\right)^{n}$.

Let $\widehat{u} \in L_{\mathcal{D}, \nu}$ such that $\|\widehat{u}\|_{L_{\mathcal{D}, \nu}}=1$, and take $(\widehat{\mathbf{v}}, \widehat{F})$ compatible with $\widehat{u}$ such that

$$
\sum_{i=1}^{d}\left\|\widehat{\mathbf{v}}^{(i)}\right\|_{L^{2}(\Omega)^{d}}^{2}+\sum_{i=1}^{d} \sum_{K \in \mathcal{M}} \sum_{\sigma \in \mathcal{E}_{K}} \nu \frac{\operatorname{diam}(K)}{\mathrm{m}(\sigma)}\left|\widehat{F}_{K, \sigma}^{(i)}\right|^{2}=1 .
$$

Define $\overrightarrow{\widehat{u}}_{\sigma}=\left(\widehat{u}_{\sigma}^{(1)}, \ldots, \widehat{u}_{\sigma}^{(d)}\right)$ as in Remark 2.3 with $(u, \mathbf{v}, \widetilde{F})=(\widehat{u}, \widehat{\mathbf{v}}, \widehat{F})$ (which satisfy (9) and (13)); we have $\sum_{\sigma \in \mathcal{E}_{K}} \mathrm{~m}(\sigma) \overrightarrow{\hat{u}}_{\sigma} \cdot \mathbf{n}_{K, \sigma}=0$.

By (26), we have

$$
\begin{aligned}
\sum_{\sigma \in \mathcal{E}_{K}} \mathrm{~m}(\sigma) \vec{u}_{\sigma}^{n+1 / 2} \cdot \mathbf{n}_{K, \sigma}( & \left.\frac{\left(u^{(i)}\right)_{K}^{n+1 / 2}+\left(u^{(i)}\right)_{L}^{n+1 / 2}}{2}\right) \\
& =\sum_{\sigma \in \mathcal{E}_{K}} \mathrm{~m}(\sigma) \vec{u}_{\sigma}^{n+1 / 2} \cdot \mathbf{n}_{K, \sigma}\left(\frac{\left(u^{(i)}\right)_{L}^{n+1 / 2}-\left(u^{(i)}\right)_{K}^{n+1 / 2}}{2}\right) .
\end{aligned}
$$


Substituting this in the second term of (21) and multiplying the resulting equation by $\delta t \widehat{u}_{K}^{(i)}$ and summing on $K \in \mathcal{M}$ and $i=1, \ldots, d$, we obtain $T_{6}=-T_{7}+T_{8}+T_{9}$ with

$$
\begin{aligned}
& T_{6}=\sum_{i=1}^{d} \sum_{K \in \mathcal{M}} \mathrm{m}(K)\left(\left(u^{(i)}\right)_{K}^{n+1}-\left(u^{(i)}\right)_{K}^{n}\right) \widehat{u}_{K}^{(i)} \\
& T_{7}=\delta t \sum_{i=1}^{d} \sum_{K \in \mathcal{M}} \sum_{\sigma \in \mathcal{E}_{K}} \mathrm{~m}(\sigma) \vec{u}_{\sigma}^{n+1 / 2} \cdot \mathbf{n}_{K, \sigma}\left(\frac{\left(u^{(i)}\right)_{L}^{n+1 / 2}-\left(u^{(i)}\right)_{K}^{n+1 / 2}}{2}\right) \widehat{u}_{K}^{(i)} \\
& T_{8}=\delta t \sum_{i=1}^{d} \sum_{K \in \mathcal{M}} \sum_{\sigma \in \mathcal{E}_{K}}\left(F^{(i)}\right)_{K, \sigma}^{n+1 / 2} \widehat{u}_{K}^{(i)} \\
& T_{9}=\sum_{i=1}^{d} \int_{n \delta t}^{(n+1) \delta t} \int_{\Omega} f^{(i)}(t, x) \widehat{u}^{(i)}(x) \mathrm{d} t \mathrm{~d} x
\end{aligned}
$$

Let us estimate $T_{7}$. We have, denoting $\vec{u}_{K}^{n+1 / 2}=\left(\left(u^{(1)}\right)_{K}^{n+1 / 2}, \ldots,\left(u^{(d)}\right)_{K}^{n+1 / 2}\right)$,

$$
\begin{aligned}
2 T_{7}= & \delta t \sum_{i=1}^{d} \sum_{K \in \mathcal{M}} \sum_{\sigma \in \mathcal{E}_{K}} \mathrm{~m}(\sigma)\left(\vec{u}_{\sigma}^{n+1 / 2}-\vec{u}_{K}^{n+1 / 2}\right) \cdot \mathbf{n}_{K, \sigma}\left(\left(u^{(i)}\right)_{L}^{n+1 / 2}-\left(u^{(i)}\right)_{K}^{n+1 / 2}\right) \widehat{u}_{K}^{(i)} \\
& +\delta t \sum_{i=1}^{d} \sum_{K \in \mathcal{M}} \sum_{\sigma \in \mathcal{E}_{K}} \mathrm{~m}(\sigma) \vec{u}_{K}^{n+1 / 2} \cdot \mathbf{n}_{K, \sigma}\left(\left(u^{(i)}\right)_{L}^{n+1 / 2}-\left(u^{(i)}\right)_{K}^{n+1 / 2}\right) \widehat{u}_{K}^{(i)} \\
= & \delta t T_{7}^{a}+\delta t T_{7}^{b} .
\end{aligned}
$$

Using Hölder's inequality with exponents 2, 4 and 4, we have

$$
\begin{aligned}
\left|T_{7}^{a}\right| \leq & \sum_{i=1}^{d} \sum_{K \in \mathcal{M}} \sum_{\sigma \in \mathcal{E}_{K}} \mathrm{~m}(\sigma) \operatorname{diam}(K) \frac{\left|\vec{u}_{\sigma}^{n+1 / 2}-\vec{u}_{K}^{n+1 / 2}\right|}{\operatorname{diam}(K)}\left|\left(u^{(i)}\right)_{L}^{n+1 / 2}-\left(u^{(i)}\right)_{K}^{n+1 / 2}\right|\left|\widehat{u}_{K}^{(i)}\right| \\
\leq & \left(\sum_{i=1}^{d} \sum_{K \in \mathcal{M}} \sum_{\sigma \in \mathcal{E}_{K}} \mathrm{~m}(\sigma) \operatorname{diam}(K) \frac{\left|\vec{u}_{\sigma}^{n+1 / 2}-\vec{u}_{K}^{n+1 / 2}\right|^{2}}{\operatorname{diam}(K)^{2}}\right)^{1 / 2} \\
& \times\left(\sum_{i=1}^{d} \sum_{K \in \mathcal{M}} \sum_{\sigma \in \mathcal{E}_{K}} \mathrm{~m}(\sigma) \operatorname{diam}(K)\left(\left|\left(u^{(i)}\right)_{L}^{n+1 / 2}\right|+\left|\left(u^{(i)}\right)_{K}^{n+1 / 2}\right|\right)^{4}\right)^{1 / 4} \\
& \times\left(\sum_{i=1}^{d} \sum_{K \in \mathcal{M}} \sum_{\sigma \in \mathcal{E}_{K}} \mathrm{~m}(\sigma) \operatorname{diam}(K)\left|\widehat{u}_{K}^{(i)}\right|^{4}\right)^{1 / 4} .
\end{aligned}
$$

The same way we estimate the discrete $H^{1}$-norm in Lemma 6.2 , it is easy to see from the definition of $\vec{u}_{\sigma}^{n+1 / 2}$ that

$$
\sum_{i=1}^{d} \sum_{K \in \mathcal{M}} \sum_{\sigma \in \mathcal{E}_{K}} \frac{\mathrm{m}(\sigma)}{\operatorname{diam}(K)}\left|\vec{u}_{\sigma}^{n+1 / 2}-\vec{u}_{K}^{n+1 / 2}\right|^{2}
$$




$$
\leq C_{8}\left(\sum_{i=1}^{d}\left\|\left(\mathbf{v}^{(i)}\right)^{n+1 / 2}\right\|_{L^{2}(\Omega)^{d}}^{2}+\sum_{i=1}^{d} \sum_{K \in \mathcal{M}} \sum_{\sigma \in \mathcal{E}_{K}} \nu^{2} \frac{\operatorname{diam}(K)}{\mathrm{m}(\sigma)}\left|\left(\widetilde{F}^{(i)}\right)_{K, \sigma}^{n+1 / 2}\right|^{2}\right)
$$

and therefore, thanks to $(8)$ and to the definition of $\operatorname{regul}(\mathcal{D})$,

$$
\begin{aligned}
\left|T_{7}^{a}\right| \leq & C_{9}\left(\sum_{i=1}^{d}\left\|\left(\mathbf{v}^{(i)}\right)^{n+1 / 2}\right\|_{L^{2}(\Omega)^{d}}^{2}+\sum_{i=1}^{d} \sum_{K \in \mathcal{M}} \sum_{\sigma \in \mathcal{E}_{K}} \nu^{2} \frac{\operatorname{diam}(K)}{\mathrm{m}(\sigma)}\left|\left(\widetilde{F}^{(i)}\right)_{K, \sigma}^{n+1 / 2}\right|^{2}\right)^{1 / 2} \\
& \times\left(\sum_{i=1}^{d}\left\|\left(u^{(i)}\right)^{n+1 / 2}\right\|_{L^{4}(\Omega)}^{4}\right)^{1 / 4}\left(\sum_{i=1}^{d}\left\|\widehat{u}^{(i)}\right\|_{L^{4}(\Omega)}^{4}\right)^{1 / 4} \cdot
\end{aligned}
$$

Using Lemma 6.2 for $\left(u^{(i)}\right)^{n+1 / 2}$ and $\widehat{u}^{(i)}$ with $q=4$, and recalling (42), we obtain

$$
\left|T_{7}^{a}\right| \leq C_{10}\left(\sum_{i=1}^{d}\left\|\left(\mathbf{v}^{(i)}\right)^{n+1 / 2}\right\|_{L^{2}(\Omega)^{d}}^{2}+\sum_{i=1}^{d} \sum_{K \in \mathcal{M}} \sum_{\sigma \in \mathcal{E}_{K}} \nu^{2} \frac{\operatorname{diam}(K)}{\mathrm{m}(\sigma)}\left|\left(\widetilde{F}^{(i)}\right)_{K, \sigma}^{n+1 / 2}\right|^{2}\right) .
$$

For $T_{7}^{b}$, we write

$$
\begin{aligned}
\left|T_{7}^{b}\right| \leq & \sum_{i=1}^{d} \sum_{K \in \mathcal{M}} \sum_{\sigma \in \mathcal{E}_{K}} \mathrm{~m}(\sigma) \operatorname{diam}(K)\left|\vec{u}_{K}^{n+1 / 2}\right|\left|\frac{\left(u^{(i)}\right)_{L}^{n+1 / 2}-\left(u^{(i)}\right)_{K}^{n+1 / 2}}{\operatorname{diam}(K)}\right|\left|\widehat{u}_{K}^{(i)}\right| \\
\leq & \left(\sum_{i=1}^{d} \sum_{K \in \mathcal{M}} \sum_{\sigma \in \mathcal{E}_{K}} \mathrm{~m}(\sigma) \operatorname{diam}(K)\left|\vec{u}_{K}^{n+1 / 2}\right|^{4}\right)^{1 / 4} \\
& \times\left(\sum_{i=1}^{d} \sum_{K \in \mathcal{M}} \sum_{\sigma \in \mathcal{E}_{K}} \mathrm{~m}(\sigma) \operatorname{diam}(K) \frac{\left|\left(u^{(i)}\right)_{L}^{n+1 / 2}-\left(u^{(i)}\right)_{K}^{n+1 / 2}\right|^{2}}{\operatorname{diam}(K)^{2}}\right)^{1 / 2} \\
& \times\left(\sum_{i=1}^{d} \sum_{K \in \mathcal{M}} \sum_{\sigma \in \mathcal{E}_{K}} \mathrm{~m}(\sigma) \operatorname{diam}(K)\left|\widehat{u}_{K}^{(i)}\right|^{4}\right)^{1 / 4}
\end{aligned}
$$

and, thanks to the estimates on the discrete $H^{1}$-norm and the $L^{4}$ norms in Lemma $6.2, T_{7}^{b}$ is estimated the same way as $T_{7}^{a}$. This finally gives, since $\nu \leq \nu_{0}$,

$$
\left|T_{7}\right| \leq C_{11} \delta t\left(\sum_{i=1}^{d}\left\|\left(\mathbf{v}^{(i)}\right)^{n+1 / 2}\right\|_{L^{2}(\Omega)^{d}}^{2}+\sum_{i=1}^{d} \sum_{K \in \mathcal{M}} \sum_{\sigma \in \mathcal{E}_{K}} \nu \frac{\operatorname{diam}(K)}{\operatorname{m}(\sigma)}\left|\left(\widetilde{F}^{(i)}\right)_{K, \sigma}^{n+1 / 2}\right|^{2}\right) .
$$

We now turn to $T_{8}$. Noting that $\widehat{u}_{\sigma}^{(i)}=0$ if $\sigma \in \mathcal{E}_{\text {ext }}$, we have $\sum_{K \in \mathcal{M}} \sum_{\sigma \in \mathcal{E}_{K}}\left(F^{(i)}\right)_{K, \sigma}^{n+1 / 2} \widehat{u}_{\sigma}^{(i)}=$ $\sum_{\sigma^{K} \mid L} \in \mathcal{E}_{\text {int }}\left(\left(F^{(i)}\right)_{K, \sigma}^{n+1 / 2}+\left(F^{(i)}\right)_{L, \sigma}^{n+1 / 2}\right) \widehat{u}_{\sigma}^{(i)}=0$ thanks to (18), and therefore, by (17),

$$
-T_{8}=\delta t \sum_{i=1}^{d} \sum_{K \in \mathcal{M}} \sum_{\sigma \in \mathcal{E}_{K}}\left(F^{(i)}\right)_{K, \sigma}^{n+1 / 2}\left(\widehat{u}_{\sigma}^{(i)}-\widehat{u}_{K}^{(i)}\right)
$$




$$
\begin{aligned}
= & \delta t \sum_{i=1}^{d} \sum_{K \in \mathcal{M}} \sum_{\sigma \in \mathcal{E}_{K}}\left(\widetilde{F}^{(i)}\right)_{K, \sigma}^{n+1 / 2}\left(\widehat{u}_{\sigma}^{(i)}-\widehat{u}_{K}^{(i)}\right) \\
& -\delta t \sum_{K \in \mathcal{M}} p_{K}^{n+1 / 2} \sum_{\sigma \in \mathcal{E}_{K}} \sum_{i=1}^{d} \mathrm{~m}(\sigma)\left(\widehat{u}_{\sigma}^{(i)}-\widehat{u}_{K}^{(i)}\right) \mathbf{e}^{(i)} \cdot \mathbf{n}_{K, \sigma} .
\end{aligned}
$$

Since $\sum_{\sigma \in \mathcal{E}_{K}} \mathrm{~m}(\sigma) \mathbf{n}_{K, \sigma}$ and $\sum_{\sigma \in \mathcal{E}_{K}} \mathrm{~m}(\sigma) \overrightarrow{\widehat{u}}_{\sigma} \cdot \mathbf{n}_{K, \sigma}$ are null, we deduce, by definition of $\widehat{u}_{\sigma}^{(i)}$,

$$
\begin{aligned}
-T_{8}= & \delta t \sum_{i=1}^{d} \sum_{K \in \mathcal{M}} \sum_{\sigma \in \mathcal{E}_{K}}\left(\widetilde{F}^{(i)}\right)_{K, \sigma}^{n+1 / 2}\left(\widehat{u}_{\sigma}^{(i)}-\widehat{u}_{K}^{(i)}\right) \\
= & \delta t \sum_{i=1}^{d} \sum_{K \in \mathcal{M}} \widehat{\mathbf{v}}_{K}^{(i)} \cdot \sum_{\sigma \in \mathcal{E}_{K}}\left(\widetilde{F}^{(i)}\right)_{K, \sigma}^{n+1 / 2}\left(\mathbf{x}_{\sigma}-\mathbf{x}_{K}\right) \\
& +\delta t \sum_{i=1}^{d} \sum_{K \in \mathcal{M}} \sum_{\sigma \in \mathcal{E}_{K}} \nu \frac{\operatorname{diam}(K)}{\mathrm{m}(\sigma)}\left(\widetilde{F}^{(i)}\right)_{K, \sigma}^{n+1 / 2} \widehat{F}_{K, \sigma}^{(i)} \\
= & \delta t \sum_{i=1}^{d} \sum_{K \in \mathcal{M}} \mathrm{m}(K) \widehat{\mathbf{v}}_{K}^{(i)} \cdot\left(\mathbf{v}^{(i)}\right)_{K}^{n+1 / 2}+\delta t \sum_{i=1}^{d} \sum_{K \in \mathcal{M}} \sum_{\sigma \in \mathcal{E}_{K}} \nu \frac{\operatorname{diam}(K)}{\mathrm{m}(\sigma)}\left(\widetilde{F}^{(i)}\right)_{K, \sigma}^{n+1 / 2} \widehat{F}_{K, \sigma}^{(i)} .
\end{aligned}
$$

The choice (42) then implies

$$
\left|T_{8}\right| \leq \delta t \sum_{i=1}^{d}\left\|\left(\mathbf{v}^{(i)}\right)^{n+1 / 2}\right\|_{L^{2}(\Omega)}+\delta t\left(\sum_{i=1}^{d} \sum_{K \in \mathcal{M}} \sum_{\sigma \in \mathcal{E}_{K}} \nu \frac{\operatorname{diam}(K)}{\mathrm{m}(\sigma)}\left|\left(\widetilde{F}^{(i)}\right)_{K, \sigma}^{n+1 / 2}\right|^{2}\right)^{1 / 2} .
$$

We apply Lemma 6.2 to $\left(\widehat{u}^{(i)}, \widehat{\mathbf{v}}^{(i)}, \widehat{F}^{(i)}\right)$ to estimate $\left\|\widehat{u}^{(i)}\right\|_{L^{2}(\Omega)}$ and, since $\nu \leq \nu_{0}$, the equation (42) gives

$$
\left|T_{9}\right| \leq C_{12} \sum_{i=1}^{d} \int_{n \delta t}^{(n+1) \delta t}\left\|f^{(i)}(t, \cdot)\right\|_{L^{2}(\Omega)} .
$$

Gathering (45), (46) and (47) in $T_{6}=-T_{7}+T_{8}+T_{9}$, and since the resulting estimate is valid for all $\widehat{u} \in L_{\mathcal{D}, \nu}$ with norm 1, we conclude, from Young's inequality, that

$$
\begin{aligned}
\left\|u^{n+1}-u^{n}\right\|_{L_{\mathcal{D}, \nu}^{*}} \leq & C_{13} \sum_{i=1}^{d} \int_{n \delta t}^{(n+1) \delta t}\left\|f^{(i)}(t, \cdot)\right\|_{L^{2}(\Omega)}+C_{13} \delta t \sum_{i=1}^{d}\left\|\left(\mathbf{v}^{(i)}\right)^{n+1 / 2}\right\|_{L^{2}(\Omega)^{d}}^{2} \\
& +C_{13} \delta t \sum_{i=1}^{d} \sum_{K \in \mathcal{M}} \sum_{\sigma \in \mathcal{E}_{K}} \nu \frac{\operatorname{diam}(K)}{\mathrm{m}(\sigma)}\left|\left(\widetilde{F}^{(i)}\right)_{K, \sigma}^{n+1 / 2}\right|^{2}+C_{13} \delta t .
\end{aligned}
$$

Step 2: conclusion.

For all $t \in] 0, T\left[, u(t, \cdot)\right.$ belongs to $L_{\mathcal{D}, \nu}\left(\right.$ since $u^{(i)}(t, \cdot)=\left(u^{(i)}\right)^{n+1 / 2}$ for some $\left.n=0, \ldots, N_{\delta t}-1\right)$. Hence, we can apply (41) and we have, by Young's inequality,

$$
\begin{aligned}
\int_{0}^{T-\tau}\|u(t+\tau, \cdot)-u(t, \cdot)\|_{L^{2}(\Omega)} \mathrm{d} t \leq & \frac{1}{2 \sqrt{\tau}} \int_{0}^{T-\tau}\|u(t+\tau, \cdot)-u(t, \cdot)\|_{L_{\mathcal{D}, \nu}^{*}} \mathrm{~d} t \\
& +\frac{\sqrt{\tau}}{2} \int_{0}^{T-\tau}\|u(t+\tau, \cdot)-u(t, \cdot)\|_{L_{\mathcal{D}, \nu}} \mathrm{d} t .
\end{aligned}
$$


But, for all $t \in] 0, T[$, denoting $n$ the integer such that $t \in] n \delta t,(n+1) \delta t\left[\right.$ (so that $u^{(i)}(t, \cdot)=$ $\left(u^{(i)}\right)^{n+1 / 2}$, we have by definition of the norm of $L_{\mathcal{D}, \nu}$,

$$
\|u(t, \cdot)\|_{L_{\mathcal{D}, \nu}}^{2} \leq \sum_{i=1}^{d}\left\|\left(\mathbf{v}^{(i)}\right)^{n+1 / 2}\right\|_{L^{2}(\Omega)^{d}}^{2}+\sum_{i=1}^{d} \sum_{K \in \mathcal{M}} \sum_{\sigma \in \mathcal{E}_{K}} \nu \frac{\operatorname{diam}(K)}{\mathrm{m}(\sigma)}\left|\left(\widetilde{F}^{(i)}\right)_{K, \sigma}^{n+1 / 2}\right|^{2}
$$

so that, by the estimates in Proposition 5.1,

$$
\begin{aligned}
\int_{0}^{T}\|u(t, \cdot)\|_{L_{\mathcal{D}, \nu}}^{2} \leq & \sum_{n=0}^{N_{\delta t}-1} \delta t \sum_{i=1}^{d}\left\|\left(\mathbf{v}^{(i)}\right)^{n+1 / 2}\right\|_{L^{2}(\Omega)^{d}}^{2} \\
& +\sum_{n=0}^{N_{\delta t}-1} \delta t \sum_{i=1}^{d} \sum_{K \in \mathcal{M}} \sum_{\sigma \in \mathcal{E}_{K}} \nu \frac{\operatorname{diam}(K)}{\mathrm{m}(\sigma)}\left|\left(\widetilde{F}^{(i)}\right)_{K, \sigma}^{n+1 / 2}\right|^{2} \leq C_{14}
\end{aligned}
$$

and (49) is reduced to

$$
\int_{0}^{T-\tau}\|u(t+\tau, \cdot)-u(t, \cdot)\|_{L^{2}(\Omega)} \mathrm{d} t \leq \frac{1}{2 \sqrt{\tau}} \int_{0}^{T-\tau}\|u(t+\tau, \cdot)-u(t, \cdot)\|_{L_{\mathcal{D}, \nu}^{*}}+C_{15} \sqrt{\tau} .
$$

For $t \in] 0, T-\tau\left[\right.$, let $n_{0}(t)$ and $n_{1}(t)$ be the integer parts of $t / \delta t$ and $(t+\tau) / \delta t$ (these integers belong to $\left.\left\{0, \ldots, N_{\delta t}-1\right\}\right)$. We have $\|u(t+\tau, \cdot)-u(t, \cdot)\|_{L_{\mathcal{D}, \nu}^{*}} \leq \sum_{n=n_{0}(t)}^{n_{1}(t)-1}\left\|u^{n+3 / 2}-u^{n+1 / 2}\right\|_{L_{\mathcal{D}, \nu}^{*}}$. By (22), $u^{n+3 / 2}-u^{n+1 / 2}=\frac{u^{n+2}+u^{n+1}}{2}-\frac{u^{n+1}+u^{n}}{2}=\frac{u^{n+2}-u^{n+1}}{2}+\frac{u^{n+1}-u^{n}}{2}$ so that, using Fubini's theorem,

$$
\begin{aligned}
\int_{0}^{T-\tau}\|u(t+\tau, \cdot)-u(t, \cdot)\|_{L_{\mathcal{D}, \nu}^{*}} \mathrm{~d} t \\
\leq \int_{0}^{T-\tau} \sum_{n=n_{0}(t)}^{n_{1}(t)-1} \frac{\left\|u^{n+2}-u^{n+1}\right\|_{L_{\mathcal{D}, \nu}^{*}}}{2}+\frac{\left\|u^{n+1}-u^{n}\right\|_{L_{\mathcal{D}, \nu}^{*}}}{2} \mathrm{~d} t \\
\leq \sum_{n=0}^{N_{\delta t}-2}\left(\frac{\left\|u^{n+2}-u^{n+1}\right\|_{L_{\mathcal{D}, \nu}^{*}}}{2}+\frac{\left\|u^{n+1}-u^{n}\right\|_{L_{\mathcal{D}, \nu}^{*}}}{2}\right) \int_{0}^{T-\tau} \chi(n, t) \mathrm{d} t
\end{aligned}
$$

where $\chi(n, t)=1$ if $n_{0}(t) \leq n \leq n_{1}(t)-1$, and 0 otherwise. We have $\chi(n, t)=1$ if and only if $n>(t / \delta t)-1$ and $n+1 \leq(t+\tau) / \delta t$, i.e. $t \in\left[(n+1) \delta t-\tau,(n+1) \delta t\left[\right.\right.$, so that $\int_{0}^{T-\tau} \chi(n, t) \mathrm{d} t \leq \tau$. By (48) and the estimates of Proposition 5.1, we obtain

$$
\begin{aligned}
\int_{0}^{T-\tau}\|u(t+\tau, \cdot)-u(t, \cdot)\|_{L_{\mathcal{D}, \nu}^{*}} \mathrm{~d} t \leq & C_{16} \tau\left(\|f\|_{L^{1}\left(0, T ; L^{2}(\Omega)\right)^{d}}+\sum_{i=1}^{d}\left\|\mathbf{v}^{(i)}\right\|_{L^{2}(] 0, T[\times \Omega)^{d}}^{2}\right. \\
& \left.+\sum_{i=1}^{d} \sum_{n=0}^{N_{\delta t}-1} \delta \sum_{K \in \mathcal{M}} \sum_{\sigma \in \mathcal{E}_{K}} \nu \frac{\operatorname{diam}(K)}{\mathrm{m}(\sigma)}\left|\left(\widetilde{F}^{(i)}\right)_{K, \sigma}^{n+1 / 2}\right|^{2}+T\right) \\
\leq & C_{17} \tau
\end{aligned}
$$

and the proof is concluded by using this estimate in (50). 


\subsection{Proof of the convergence}

We now prove Theorem 5.2. To simplify the notations, we drop the index $m$ and we study the convergence of $u=\left(u^{(1)}, \ldots, u^{(d)}\right)$ and $\mathbf{v}=\left(\mathbf{v}^{(1)}, \ldots, \mathbf{v}^{(d)}\right)$ as $\operatorname{size}(\mathcal{D}) \rightarrow 0$ and $\delta t \rightarrow 0$ while $\operatorname{regul}(\mathcal{D})$ remains bounded.

Proposition 5.1 gives estimates, independent on $\mathcal{D}$ or $\delta t$, on $\left(u^{(i)}, \mathbf{v}^{(i)}, \widetilde{F}^{(i)}\right)_{i=1, \ldots, d}$. Recalling the definition of $N_{2}$ from Lemma 6.2, these estimates show that

$$
\sum_{i=1}^{d} \sum_{n=0}^{N_{\delta t}-1} \delta t N_{2}\left(\mathcal{D}, \nu,\left(\widetilde{F}^{(i)}\right)^{n+1 / 2}\right)^{2}=\sum_{i=1}^{d} \sum_{n=0}^{N_{\delta t}-1} \delta t \sum_{K \in \mathcal{M}} \sum_{\sigma \in \mathcal{E}_{K}} \nu^{2} \frac{\operatorname{diam}(K)}{\mathrm{m}(\sigma)}\left|\left(\widetilde{F}^{(i)}\right)_{K, \sigma}^{n+1 / 2}\right|^{2} \rightarrow 0
$$

as $\operatorname{size}(\mathcal{D}) \rightarrow 0$ (recall that $\nu=\lambda \operatorname{size}(\mathcal{D})^{\alpha}$ with $\left.\alpha \in\right] 0,2[$ ). In particular, applying Lemma 6.2 to $\left(\left(u^{(i)}\right)^{n+1 / 2},\left(\mathbf{v}^{(i)}\right)^{n+1 / 2},\left(\widetilde{F}^{(i)}\right)^{n+1 / 2}\right)$, taking the square of the resulting estimate, multiplying by $\delta t$ and summing on $n=0, \ldots, N_{\delta t}-1$, we see that $u$ is bounded in $L^{2}\left(0, T ; L^{q}(\Omega)\right)^{d}$ for all $q<+\infty$ if $d=2$ and all $q \leq 6$ if $d=3$. Since $u$ is also bounded in $L^{\infty}\left(0, T ; L^{2}(\Omega)\right)^{d}$ (Proposition 5.1 , we deduce by interpolation that it is bounded in $L^{2+\epsilon}(] 0, T[\times \Omega)^{d}$ for some $\epsilon>0$.

By Cauchy-Schwarz inequality, (8) and (51), we have

$$
\begin{aligned}
& \sum_{i=1}^{d} \sum_{n=0}^{N_{\delta t}-1} \delta t \sum_{K \in \mathcal{M}} \sum_{\sigma \in \mathcal{E}_{K}} \nu \operatorname{diam}(K)\left|\left(\widetilde{F}^{(i)}\right)_{K, \sigma}^{n+1 / 2}\right| \\
& \quad \leq C_{18}\left(\sum_{i=1}^{d} \sum_{n=0}^{N_{\delta t}-1} \delta t \sum_{K \in \mathcal{M}} \sum_{\sigma \in \mathcal{E}_{K}} \nu^{2} \frac{\operatorname{diam}(K)}{\mathrm{m}(\sigma)}\left|\left(\widetilde{F}^{(i)}\right)_{K, \sigma}^{n+1 / 2}\right|^{2}\right)^{1 / 2} \rightarrow 0 \quad \text { as size }(\mathcal{D}) \rightarrow 0
\end{aligned}
$$

$\left(C_{18}\right.$ does not depend on $\mathcal{D}$ or $\left.\delta t\right)$. In particular, taking the estimate given by Lemma 6.3 applied to $\left(\left(u^{(i)}\right)^{n+1 / 2},\left(\mathbf{v}^{(i)}\right)^{n+1 / 2},\left(\widetilde{F}^{(i)}\right)^{n+1 / 2}\right)$, multiplying it by $\delta t$ and summing on $n=0, \ldots, N_{\delta t}-1$ and $i=1, \ldots, d$, we see that $\|u(\cdot, \cdot+\xi)-u\|_{L^{1}(] 0, T\left[\times \mathbb{R}^{d}\right)^{d}} \rightarrow 0$ as $|\xi| \rightarrow 0$, independently on $\mathcal{D}$ or $\delta t$ (we have extended $u$ in space by 0 outside $\Omega$ ). Proposition 5.2 gives a similar estimate on the translations in time, which therefore proves that $u$ is relatively compact in $L_{\text {loc }}^{1}(] 0, T[\times \Omega)^{d}$. Since it is bounded in $L^{2+\epsilon}(] 0, T[\times \Omega)^{d}$ for some $\epsilon>0, u$ is also relatively compact in $L^{2}(] 0, T[\times \Omega)^{d}$. Up to a subsequence, we can thus assume that $u \rightarrow \bar{u}$ strongly in $L^{2}(] 0, T[\times \Omega)^{d}$ and that $\mathbf{v}$ weakly converges in $L^{2}(] 0, T[\times \Omega)^{d \times d}$. It is then easy to deduce from (51) that the weak limit of $\mathbf{v}$ is $\nabla \bar{u}$ and that $\bar{u} \in L^{2}\left(0, T ; H_{0}^{1}(\Omega)\right)^{d}$ (this is similar to the result of Lemma 6.4 ; see the proof of [6, Lemma 3.3] or the proof of [4, Lemma 7.4] for an example in a transient case).

Step 1: we prove that $\operatorname{div}(\bar{u})=0$.

Let $\Gamma:] 0, T[\times \Omega \rightarrow \mathbb{R}$ be the piecewise constant function equal, on $] n \delta t,(n+1) \delta t[\times K$, to $\frac{\nu \operatorname{diam}(K)}{\mathrm{m}(K)} \sum_{i=1}^{d} \sum_{\sigma \in \mathcal{E}_{K}}\left(\widetilde{F}^{(i)}\right)_{K, \sigma}^{n+1 / 2} \mathbf{e}^{(i)} \cdot \mathbf{n}_{K, \sigma}$. Thanks to (52), we have $\Gamma \rightarrow 0$ in $L^{1}(] 0, T[\times \Omega)$ as $\operatorname{size}(\mathcal{D}) \rightarrow 0$. Since $\sum_{i=1}^{d} \mathbf{v}^{(i)} \cdot \mathbf{e}^{(i)}+\Gamma=0$ (this is (20) divided by $\mathrm{m}(K)$ ), we deduce from the weak convergence of $\mathbf{v}^{(i)}$ toward $\nabla \bar{u}^{(i)}$ that $\operatorname{div}(\bar{u})=\sum_{i=1}^{d} \nabla \bar{u}^{(i)} \cdot \mathbf{e}^{(i)}=0$.

Step 2: we prove that $\bar{u}$ satisfies $(7)$.

Let $\varphi \in C_{c}^{\infty}\left(\left[0, T[\times \Omega)^{d}\right.\right.$ such that $\operatorname{div}(\varphi)=0$. We use (43) to transform the second term of (21), multiply the resulting equation by $\delta t \varphi^{(i)}\left(n \delta t, \mathbf{x}_{K}\right)$ and sum on $i, K$ and $n$. This gives $T_{10}+T_{11}+T_{12}=T_{13}$ with

$$
T_{10}=\sum_{i=1}^{d} \sum_{n=0}^{N_{\delta t}-1} \sum_{K \in \mathcal{M}} \mathrm{m}(K)\left(\left(u^{(i)}\right)_{K}^{n+1}-\left(u^{(i)}\right)_{K}^{n}\right) \varphi^{(i)}\left(n \delta t, \mathbf{x}_{K}\right)
$$




$$
\begin{aligned}
& T_{11}=\sum_{i=1}^{d} \sum_{n=0}^{N_{\delta t}-1} \delta t \sum_{K \in \mathcal{M}} \sum_{\sigma \in \mathcal{E}_{K}} \mathrm{~m}(\sigma) \vec{u}_{\sigma}^{n+1 / 2} \cdot \mathbf{n}_{K, \sigma}\left(\frac{\left(u^{(i)}\right)_{L}^{n+1 / 2}-\left(u^{(i)}\right)_{K}^{n+1 / 2}}{2}\right) \varphi^{(i)}\left(n \delta t, \mathbf{x}_{K}\right), \\
& T_{12}=-\sum_{n=0}^{N_{\delta t}-1} \delta t \sum_{i=1}^{d} \sum_{K \in \mathcal{M}} \sum_{\sigma \in \mathcal{E}_{K}}\left(F^{(i)}\right)_{K, \sigma}^{n+1 / 2} \varphi^{(i)}\left(n \delta t, \mathbf{x}_{K}\right), \\
& T_{13}=\sum_{i=1}^{d} \sum_{n=0}^{N_{\delta t}-1} \int_{n \delta t}^{(n+1) \delta t} f^{(i)}(t, x) \mathrm{d} t \mathrm{~d} x \varphi^{(i)}\left(n \delta t, \mathbf{x}_{K}\right) .
\end{aligned}
$$

We now study the convergence of each of these terms as $\operatorname{size}(\mathcal{D}) \rightarrow 0$ and $\delta t \rightarrow 0$.

Since $\varphi=0$ on a neighborhood of $t=T$, for $\delta t$ small enough we have

$$
\begin{aligned}
T_{10}= & \sum_{i=1}^{d} \sum_{n=1}^{N_{\delta t}-1} \delta t \sum_{K \in \mathcal{M}} \mathrm{m}(K)\left(u^{(i)}\right)_{K}^{n} \frac{\varphi^{(i)}\left((n-1) \delta t, \mathbf{x}_{K}\right)-\varphi^{(i)}\left(n \delta t, \mathbf{x}_{K}\right)}{\delta t} \\
& -\sum_{i=1}^{d} \sum_{K \in \mathcal{M}} \mathrm{m}(K)\left(u^{(i)}\right)_{K}^{0} \varphi^{(i)}\left(0, \mathbf{x}_{K}\right) .
\end{aligned}
$$

By (24) and regularity of $\varphi$, the second term of this right-hand side tends to $-\int_{\Omega} u_{0}(x) \cdot \varphi(0, x) \mathrm{d} x$ as $\operatorname{size}(\mathcal{D}) \rightarrow 0$. Let $\left.\Pi^{(i)}:\right] 0, T\left[\times \Omega \rightarrow \mathbb{R}\right.$ and $\left.U^{(i)}:\right] 0, T[\times \Omega \rightarrow \mathbb{R}$ be the piecewise functions respectively equal to $\frac{\varphi^{(i)}\left((n-1) \delta t, \mathbf{x}_{K}\right)-\varphi^{(i)}\left(n \delta t, \mathbf{x}_{K}\right)}{\delta t}$ and $\left(u^{(i)}\right)_{K}^{n}$ on $] n \delta t,(n+1) \delta t[\times K$ for all $n=$ $0, \ldots, N_{\delta t}-1$ and all $K \in \mathcal{M}$. We have

$$
T_{10}=\sum_{i=1}^{d} \int_{0}^{T} \int_{\Omega} U^{(i)}(t, x) \Pi^{(i)}(t, x) \mathrm{d} t \mathrm{~d} x-\int_{\Omega} u_{0}(x) \cdot \varphi(0, x) \mathrm{d} x+\zeta(\mathcal{D})
$$

where $\zeta(\mathcal{D}) \rightarrow 0$ as $\operatorname{size}(\mathcal{D}) \rightarrow 0$. The regularity of $\varphi$ ensures that $\Pi^{(i)} \rightarrow-\partial_{t} \varphi^{(i)}$ uniformly on ] $0, T\left[\times \Omega\right.$. The estimate (40) shows that $U^{(i)}$ is bounded in $L^{\infty}\left(0, T ; L^{2}(\Omega)\right)$ and therefore that, up to a subsequence, it converges to some $\bar{U}^{(i)}$ weakly-* in this space. But, on $] 0, T-\delta t[\times \Omega$, (22) states that $u^{(i)}(t, x)=\frac{U^{(i)}(t+\delta t, x)+U^{(i)}(t, x)}{2}$ and it is then not difficult to see that $u^{(i)}$ must also converge weakly-* in $L^{\infty}\left(0, T ; L^{2}(\Omega)\right)$ to $\bar{U}^{(i)}$; hence, $\bar{U}^{(i)}=\bar{u}^{(i)}$ and we can pass to the limit in (53) to find

$$
T_{10} \rightarrow-\sum_{i=1}^{d} \int_{0}^{T} \int_{\Omega} \bar{u}^{(i)}(t, x) \partial_{t} \varphi^{(i)}(t, x) \mathrm{d} t \mathrm{~d} x-\int_{\Omega} u_{0}(x) \cdot \varphi(0, x) \mathrm{d} x
$$

as $\operatorname{size}(\mathcal{D}) \rightarrow 0$ and $\delta t \rightarrow 0$.

Gathering $T_{11}$ by edges, we have, for $\operatorname{size}(\mathcal{D})$ small enough (so that $\varphi=0$ on the boundary control volumes),

$$
\begin{aligned}
T_{11}=\sum_{i=1}^{d} \sum_{n=0}^{N_{\delta t}-1} \delta t \sum_{\sigma^{K \mid L} \in \mathcal{E}_{\mathrm{int}}} \mathrm{m}(\sigma) & \vec{u}_{\sigma}^{n+1 / 2} \cdot \mathbf{n}_{K, \sigma} \\
& \times\left(\left(u^{(i)}\right)_{L}^{n+1 / 2}-\left(u^{(i)}\right)_{K}^{n+1 / 2}\right) \frac{\varphi^{(i)}\left(n \delta t, \mathbf{x}_{K}\right)+\varphi^{(i)}\left(n \delta t, \mathbf{x}_{L}\right)}{2} .
\end{aligned}
$$


Let $\diamond$ be a $\mathcal{D}$-adapted diamond partition of $\Omega$ according to Definition 6.1. We have

$$
\begin{aligned}
T_{11}=\sum_{i=1}^{d} \sum_{n=0}^{N_{\delta t}-1} \delta t & \sum_{\sigma^{K \mid L} \in \mathcal{E}_{\mathrm{int}}} \mathrm{m}\left(\diamond_{\sigma}\right) \frac{\varphi^{(i)}\left(n \delta t, \mathbf{x}_{K}\right)+\varphi^{(i)}\left(n \delta t, \mathbf{x}_{L}\right)}{2} \\
& \times \vec{u}_{\sigma}^{n+1 / 2} \cdot \frac{\mathrm{m}(\sigma)}{\mathrm{m}\left(\diamond_{\sigma}\right)}\left(\left(u^{(i)}\right)_{L}^{n+1 / 2}-\left(u^{(i)}\right)_{K}^{n+1 / 2}\right) \mathbf{n}_{K, \sigma} .
\end{aligned}
$$

Denoting $\Psi^{(i)}, u_{\diamond}$ and $V_{\diamond}^{(i)}$ the functions respectively equal to $\frac{\varphi^{(i)}\left(n \delta t, \mathbf{x}_{K}\right)+\varphi^{(i)}\left(n \delta t, \mathbf{x}_{L}\right)}{2}, \vec{u}_{\sigma}^{n+1 / 2}$ and $\frac{\mathrm{m}(\sigma)}{\mathrm{m}\left(\diamond_{\sigma}\right)}\left(\left(u^{(i)}\right)_{L}^{n+1 / 2}-\left(u^{(i)}\right)_{K}^{n+1 / 2}\right) \mathbf{n}_{K, \sigma}$ on $] n \delta t,(n+1) \delta t\left[\times \diamond_{\sigma}\right.$, we see that

$$
T_{11}=\sum_{i=1}^{d} \int_{0}^{T} \int_{\Omega} \Psi^{(i)}(t, x) u_{\diamond}(t, x) \cdot V_{\diamond}^{(i)}(t, x) \mathrm{d} t \mathrm{~d} x .
$$

We clearly have, by regularity of $\varphi, \Psi^{(i)} \rightarrow \varphi^{(i)}$ uniformly on $] 0, T[\times \Omega$ as $\operatorname{size}(\mathcal{D}) \rightarrow 0$ and $\delta t \rightarrow 0$. From the estimates of Proposition 5.1 and Lemma 6.2, we see that $\sum_{n=0}^{N_{\delta t}-1} \delta t\left\|\left(u^{(i)}\right)^{n+1 / 2}\right\|_{1, \mathcal{D}}^{2}$ remains bounded and Lemma 6.5 therefore shows that $V_{\diamond}^{(i)} \rightarrow \nabla \bar{u}^{(i)}$ weakly in $L^{2}(] 0, T[\times \Omega)^{d}$. It is not very difficult to see that $u \diamond \rightarrow \bar{u}$ strongly in $L^{2}(] 0, T[\times \Omega)^{d}$; indeed, letting $\vec{u}_{K}^{n+1 / 2}=$ $\left(\left(u^{(1)}\right)_{K}^{n+1 / 2}, \ldots,\left(u^{(d)}\right)_{K}^{n+1 / 2}\right)$, we have

$$
\left\|u_{\diamond}-u\right\|_{L^{2}(] 0, T[\times \Omega)^{d}}=\sum_{n=0}^{N_{\delta t}-1} \delta t \sum_{K \in \mathcal{M}} \sum_{\sigma \in \mathcal{E}_{K}} \mathrm{~m}\left(\triangle_{K, \sigma}\right)\left|\vec{u}_{\sigma}^{n+1 / 2}-\vec{u}_{K}^{n+1 / 2}\right|^{2}
$$

where $\triangle_{K, \sigma}=\operatorname{co}\left(\left\{\mathbf{p}_{K}\right\} \cup \sigma\right)$ ( $\mathbf{p}_{K}$ being the point chosen to define $\diamond$, see Definition 6.1). But $\mathrm{m}\left(\triangle_{K, \sigma}\right) \leq \mathrm{m}(\sigma) \operatorname{diam}(K)$ so that, by (44) and the estimates in Proposition 5.1, the preceding quantity is bounded by $C_{19} \operatorname{size}(\mathcal{D})^{2}$ (with $C_{19}$ not depending on $\mathcal{D}$ or $\delta t$ ), and tends to 0 as $\operatorname{size}(\mathcal{D}) \rightarrow 0$. Since $u \rightarrow \bar{u}$ strongly in $L^{2}(] 0, T[\times \Omega)^{d}$, so does $u_{\diamond}$. We can thus pass to the limit in $(55)$ and we find

$$
T_{11} \rightarrow \sum_{i=1}^{d} \int_{0}^{T} \int_{\Omega} \varphi^{(i)}(t, x) \bar{u}(t, x) \cdot \nabla \bar{u}^{(i)}(t, x) \mathrm{d} t \mathrm{~d} x
$$

as $\operatorname{size}(\mathcal{D}) \rightarrow 0$ and $\delta t \rightarrow 0$.

To handle $T_{12}$, we use the same technique as for the Stokes equation. Introducing $\left(\varphi_{\sigma}^{(i)}\right)^{n}=$ $\frac{1}{\mathrm{~m}(\sigma)} \int_{\sigma} \varphi^{(i)}(n \delta t, x) \mathrm{d} \gamma(x)$ and using the fact that $\operatorname{div}(\varphi)=0$, we see (the same way we arrived at $(35))$ that

$$
T_{12}=\sum_{i=1}^{d} \int_{0}^{T} \int_{\Omega} \mathbf{v}^{(i)}(t, x) \cdot\left(\nabla \varphi^{(i)}\right)_{\delta t}(t, x) \mathrm{d} t \mathrm{~d} x+T_{14}
$$

where $\left(\nabla \varphi^{(i)}\right)_{\delta t}(\cdot, x)=\nabla \varphi^{(i)}(n \delta t, x)$ on $] n \delta t,(n+1) \delta t[$ and

$$
\left|T_{14}\right| \leq C_{\varphi} \sum_{n=0}^{N_{\delta t}-1} \delta t \sum_{i=1}^{d} \sum_{K \in \mathcal{M}} \sum_{\sigma \in \mathcal{E}_{K}}\left|\left(\widetilde{F}^{(i)}\right)_{K, \sigma}^{n+1 / 2}\right| \operatorname{diam}(K)^{2} .
$$


Using Proposition 5.1, we can show (as we did for $T_{1}$ ) that $T_{14} \rightarrow 0$ and therefore, by weak convergence of $\mathbf{v}^{(i)}$ to $\nabla \bar{u}^{(i)}$ and uniform convergence of $\left(\nabla \varphi^{(i)}\right)_{\delta t}$ to $\nabla \varphi^{(i)}$,

$$
T_{12} \rightarrow \sum_{i=1}^{d} \int_{0}^{T} \int_{\Omega} \nabla \bar{u}^{(i)}(t, x) \cdot \nabla \varphi^{(i)}(t, x) \mathrm{d} t \mathrm{~d} x
$$

as $\operatorname{size}(\mathcal{D}) \rightarrow 0$ and $\delta t \rightarrow 0$.

The convergence $T_{13} \rightarrow \int_{0}^{T} \int_{\Omega} f(t, x) \cdot \varphi(t, x) \mathrm{d} t \mathrm{~d} x$ is obvious and we conclude, from $T_{10}+T_{11}+$ $T_{12}=T_{13}$ and using (54), (56) and (57), that $\bar{u}$ satisfies (7).

\section{Appendix}

\subsection{A key relation}

The following lemma is a simple application of Stokes' formula. Its proof can be found in [6] (in the case of convex control volumes and convex edges, but the proof does not use these convexity assumptions).

Lemma 6.1 Let $K$ be a non empty open polygonal set in $\mathbb{R}^{d}$. For $\sigma \in \mathcal{E}_{K}$ (the edges of $K$, in the sense given in Definition 2.1), we let $\mathbf{x}_{\sigma}$ be the center of gravity of $\sigma$; we also denote $\mathbf{n}_{K, \sigma}$ the unit normal to $\sigma$ outward to $K$. Then, for all vector $\mathbf{e} \in \mathbb{R}^{d}$ and for all point $\mathbf{x}_{K} \in \mathbb{R}^{d}$, we have $\mathrm{m}(K) \mathbf{e}=\sum_{\sigma \in \mathcal{E}_{K}} \mathrm{~m}(\sigma) \mathbf{e} \cdot \mathbf{n}_{K, \sigma}\left(\mathbf{x}_{\sigma}-\mathbf{x}_{K}\right)$.

\subsection{The discretization space}

Assume (2) and let $\mathcal{D}$ be an admissible discretization of $\Omega$ in the sense of Definition 2.1. Let $\nu \geq 0$. We give here some properties on the $(u, \mathbf{v}, \widetilde{F}) \in H_{\mathcal{D}} \times H_{\mathcal{D}}^{d} \times \mathcal{F}_{\mathcal{D}}$ which satisfy

$$
\begin{aligned}
& \mathbf{v}_{K} \cdot\left(\mathbf{x}_{\sigma}-\mathbf{x}_{K}\right)+\mathbf{v}_{L} \cdot\left(\mathbf{x}_{L}-\mathbf{x}_{\sigma}\right)+\nu \frac{\operatorname{diam}(K)}{\mathrm{m}(\sigma)} \widetilde{F}_{K, \sigma}-\nu \frac{\operatorname{diam}(K)}{\mathrm{m}(\sigma)} \widetilde{F}_{L, \sigma}=u_{L}-u_{K}, \\
& \forall \sigma^{K \mid L} \in \mathcal{E}_{\text {int }}, \\
& \mathbf{v}_{K} \cdot\left(\mathbf{x}_{\sigma}-\mathbf{x}_{K}\right)+\nu \frac{\operatorname{diam}(K)}{\mathrm{m}(\sigma)} \widetilde{F}_{K, \sigma}=-u_{K}, \quad \forall \sigma^{K \mid \partial} \in \mathcal{E}_{\text {ext }} .
\end{aligned}
$$

In order to state those properties, we need to introduce (and in fact estimate) the following discrete $H^{1}$-norm, defined for all $u \in H_{\mathcal{D}}$ as

$$
\|u\|_{1, \mathcal{D}}=\left(\sum_{\sigma^{K \mid L} \in \mathcal{E}_{\mathrm{int}}}\left(\frac{\mathrm{m}(\sigma)}{\operatorname{diam}(K)}+\frac{\mathrm{m}(\sigma)}{\operatorname{diam}(L)}\right)\left|u_{K}-u_{L}\right|^{2}+\sum_{\sigma^{K \mid \partial \in \mathcal{E}_{\text {ext }}}} \frac{\mathrm{m}(\sigma)}{\operatorname{diam}(K)}\left|u_{K}\right|^{2}\right)^{1 / 2} .
$$

Lemma 6.2 [Estimate on the discrete $H^{1}$ norm and Sobolev inequalities] Assume that (2) holds. Let $\mathcal{D}$ be an admissible discretization of $\Omega$ in the sense of Definition 2.1 and let $\theta \geq \operatorname{regul}(\mathcal{D})$. Then there exists $C_{20}$ only depending on $d, \Omega$ and $\theta$ such that, for all $\nu \geq 0$ and all $(u, \mathbf{v}, \widetilde{F})$ satisfying (58), denoting $N_{2}(\mathcal{D}, \nu, \widetilde{F})=\left(\sum_{K \in \mathcal{M}} \sum_{\sigma \in \mathcal{E}_{K}} \nu^{2} \frac{\operatorname{diam}(K)}{\mathrm{m}(\sigma)}\left|\widetilde{F}_{K, \sigma}\right|^{2}\right)^{1 / 2}$, we have

$$
\|u\|_{1, \mathcal{D}} \leq C_{20}\left(\|\mathbf{v}\|_{L^{2}(\Omega)^{d}}+N_{2}(\mathcal{D}, \nu, \widetilde{F})\right)
$$


and, for all $q<\frac{2 d}{d-2}=+\infty$ if $d=2$ and all $q \leq \frac{2 d}{d-2}=6$ if $d=3$,

$$
\|u\|_{L^{q}(\Omega)} \leq C_{20} q\left(\|\mathbf{v}\|_{L^{2}(\Omega)^{d}}+N_{2}(\mathcal{D}, \nu, \widetilde{F})\right) .
$$

Proof of Lemma 6.2

The relations (58) show that

$$
\begin{aligned}
\|u\|_{1, \mathcal{D}}^{2} \leq & 4 \sum_{\sigma^{K \mid L} \in \mathcal{E}_{\text {int }}}\left(\frac{\mathrm{m}(\sigma)}{\operatorname{diam}(K)}+\frac{\mathrm{m}(\sigma)}{\operatorname{diam}(L)}\right)\left(\operatorname{diam}(K)^{2}\left|\mathbf{v}_{K}\right|^{2}+\operatorname{diam}(L)^{2}\left|\mathbf{v}_{L}\right|^{2}\right) \\
& +4 \sum_{\sigma^{K \mid L} \in \mathcal{E}_{\text {int }}}\left(\frac{\mathrm{m}(\sigma)}{\operatorname{diam}(K)}+\frac{\mathrm{m}(\sigma)}{\operatorname{diam}(L)}\right) \nu^{2}\left(\frac{\operatorname{diam}(K)^{2}}{\mathrm{~m}(\sigma)^{2}}\left|\widetilde{F}_{K, \sigma}\right|^{2}+\frac{\operatorname{diam}(L)^{2}}{\mathrm{~m}(\sigma)^{2}}\left|\widetilde{F}_{L, \sigma}\right|^{2}\right) \\
& +2 \sum_{\sigma^{K \mid \partial} \in \mathcal{E}_{\text {ext }}} \frac{\mathrm{m}(\sigma)}{\operatorname{diam}(K)} \operatorname{diam}(K)^{2}\left|\mathbf{v}_{K}\right|^{2}+2 \sum_{\sigma^{K \mid \partial} \in \mathcal{E}_{\text {ext }}} \frac{\mathrm{m}(\sigma)}{\operatorname{diam}(K)} \nu^{2} \frac{\operatorname{diam}(K)^{2}}{\mathrm{~m}(\sigma)^{2}}\left|\widetilde{F}_{K, \sigma}\right|^{2} .
\end{aligned}
$$

The definition of $\operatorname{regul}(\mathcal{D})$ and its bound by $\theta$ ensure that, if $K$ and $L$ are neighboring control volumes, $\operatorname{diam}(K) \leq \theta \operatorname{diam}(L)$ (and vice-versa). Hence, gathering by control volumes,

$$
\|u\|_{1, \mathcal{D}}^{2} \leq 4 \sum_{K \in \mathcal{M}}\left|\mathbf{v}_{K}\right|^{2} \sum_{\sigma \in \mathcal{E}_{K}} \mathrm{~m}(\sigma) \operatorname{diam}(K)(1+\theta)+4 \sum_{K \in \mathcal{M}} \sum_{\sigma \in \mathcal{E}_{K}} \nu^{2}\left|\widetilde{F}_{K, \sigma}\right|^{2} \frac{\operatorname{diam}(K)}{\mathrm{m}(\sigma)}(1+\theta) .
$$

By (8), the proof of (60) is complete.

Let us prove (61) in the case $d=2$. It is shown, in the proof of Lemma 9.5 in [7, p. 792] (using no assumption on the discretization of $\Omega$ ), that, for all $\alpha>1$,

$$
\left(\int_{\Omega}|u(x)|^{2 \alpha} \mathrm{d} x\right)^{\frac{1}{2}} \leq \alpha \sum_{K \in \mathcal{M}} \sum_{\sigma \in \mathcal{E}_{K}} \mathrm{~m}(\sigma)\left|u_{K}\right|^{\alpha-1} D_{\sigma} u
$$

where $D_{\sigma} u=\left|u_{K}-u_{L}\right|$ if $\sigma^{K \mid L} \in \mathcal{E}_{\text {int }}$ and $D_{\sigma} u=\left|u_{K}\right|$ if $\sigma^{K \mid \partial} \in \mathcal{E}_{\text {ext }}$. Hölder's inequality with $p=\frac{2 \alpha}{\alpha-1}>2$ and $p^{\prime}=\frac{p}{p-1}$ then gives

$$
\begin{aligned}
\left(\int_{\Omega}|u(x)|^{2 \alpha} \mathrm{d} x\right)^{\frac{1}{2}} \leq & \alpha\left(\sum_{K \in \mathcal{M}} \sum_{\sigma \in \mathcal{E}_{K}} \mathrm{~m}(\sigma) \operatorname{diam}(K)\left|u_{K}\right|^{p(\alpha-1)}\right)^{\frac{1}{p}} \\
& \times\left(\sum_{K \in \mathcal{M}} \sum_{\sigma \in \mathcal{E}_{K}} \mathrm{~m}(\sigma) \operatorname{diam}(K) \frac{\left|D_{\sigma} u\right|^{p^{\prime}}}{\operatorname{diam}(K)^{p^{\prime}}}\right)^{\frac{1}{p^{\prime}}} .
\end{aligned}
$$

Since $p(\alpha-1)=2 \alpha,(8)$ shows that

$$
\left(\int_{\Omega}|u(x)|^{2 \alpha} \mathrm{d} x\right)^{\frac{1}{2}} \leq C_{21} \alpha\left(\int_{\Omega}|u(x)|^{2 \alpha} \mathrm{d} x\right)^{\frac{1}{2}-\frac{1}{2 \alpha}}\left(\sum_{K \in \mathcal{M}} \sum_{\sigma \in \mathcal{E}_{K}} \mathrm{~m}(\sigma) \operatorname{diam}(K) \frac{\left|D_{\sigma} u\right|^{p^{\prime}}}{\operatorname{diam}(K)^{p^{\prime}}}\right)^{\frac{1}{p^{\prime}}}
$$


where $C_{21}$ only depends on $d$ and $\theta$. Since $p^{\prime}<2$, we can apply Hölder's inequality with exponent $2 / p^{\prime}$ to find, thanks again to $(8)$,

$$
\begin{aligned}
\|u\|_{L^{2 \alpha}(\Omega)} & \leq C_{21} \alpha\left(\sum_{K \in \mathcal{M}} \sum_{\sigma \in \mathcal{E}_{K}} \mathrm{~m}(\sigma) \operatorname{diam}(K)\right)^{\frac{2-p^{\prime}}{2 p^{\prime}}}\left(\sum_{K \in \mathcal{M}} \sum_{\sigma \in \mathcal{E}_{K}} \mathrm{~m}(\sigma) \operatorname{diam}(K) \frac{\left|D_{\sigma} u\right|^{2}}{\operatorname{diam}(K)^{2}}\right)^{\frac{1}{2}} \\
& \leq C_{21} \alpha\left(\frac{\omega_{d-1} \theta^{2}}{\omega_{d}} \mathrm{~m}(\Omega)\right)^{\frac{2-p^{\prime}}{2 p^{\prime}}}\left(\sum_{K \in \mathcal{M}} \sum_{\sigma \in \mathcal{E}_{K}} \frac{\mathrm{m}(\sigma)}{\operatorname{diam}(K)}\left|D_{\sigma} u\right|^{2}\right)^{\frac{1}{2}} .
\end{aligned}
$$

Gathering the last sum by edges, we recognize the discrete $H^{1}$-norm $\|u\|_{1, \mathcal{D}}$ of $u$ and (61) for $q=2 \alpha>2$ is therefore a consequence of (60); the case $1 \leq q \leq 2$ is immediate from the case $q>2$ using Hölder's inequality.

To prove (61) in the case $d=3$, we still use an inequality shown in the proof of Lemma 9.5 in [7, p. 792]:

$$
\int_{\Omega}|u(x)|^{6} \mathrm{~d} x \leq\left(4 \sum_{K \in \mathcal{M}} \sum_{\sigma \in \mathcal{E}_{K}} \mathrm{~m}(\sigma)\left|u_{K}\right|^{3} D_{\sigma} u\right)^{3 / 2} .
$$

Using Cauchy-Schwarz inequality and (8), we deduce

$$
\int_{\Omega}|u(x)|^{6} \mathrm{~d} x \leq C_{22}\left(\int_{\Omega}|u(x)|^{6} \mathrm{~d} x\right)^{3 / 4}\left(\sum_{K \in \mathcal{M}} \sum_{\sigma \in \mathcal{E}_{K}} \mathrm{~m}(\sigma) \operatorname{diam}(K) \frac{\left|D_{\sigma} u\right|^{2}}{\operatorname{diam}(K)^{2}}\right)^{3 / 4},
$$

with $C_{22}$ only depending on $d$ and $\theta$. Since the last term (involving $D_{\sigma} u$ ) is $\|u\|_{1, \mathcal{D}}^{3 / 2}$, (60) concludes the proof of (61) for $d=3$ and $q=6$; the cases $1 \leq q \leq 6$ can be deduced from the case $q=6$ thanks to Hölder's inequality.

Although the cases $d=1$ and $d \geq 4$ are not useful to us, we can notice that the Sobolev injections (61) are also valid for $d=1$ (with $q=+\infty$ ) and $d \geq 4$ (with $q \leq \frac{2 d}{d-2}$ ); the proof is made by induction on $d$ (see [7, Lemma 9.5] for $d=2$ and $d=3$ )

The two following lemmas are similar to [6, Lemmas 3.2 and 3.3], the only differences being that, in [6], the fluxes are not penalized the same way as in (58) and that we use the result of Lemma 6.2 to improve the convergence of $u_{m}$ in Lemma 6.4. We let the reader check that the proofs of these lemmas are straightforward adaptations of the proofs in [6].

Lemma 6.3 [Equicontinuity of the translations] Assume that (2) holds. Let $\mathcal{D}$ be an admissible discretization of $\Omega$ in the sense of Definition 2.1 and let $\theta \geq \operatorname{regul}(\mathcal{D})$. Let $\nu \geq 0$. Then there exists $C_{23}$ only depending on $d, \Omega$ and $\theta$ such that, for all $(u, \mathbf{v}, \widetilde{F})$ satisfying (58) and all $\xi \in \mathbb{R}^{d}$,

$$
\|u(\cdot+\xi)-u\|_{L^{1}\left(\mathbb{R}^{d}\right)} \leq C_{23}\left(\|\mathbf{v}\|_{L^{1}(\Omega)^{d}}+N_{1}(\mathcal{D}, \nu, \widetilde{F})\right)|\xi|
$$

where $N_{1}(\mathcal{D}, \nu, \widetilde{F})=\sum_{K \in \mathcal{M}} \sum_{\sigma \in \mathcal{E}_{K}} \nu \operatorname{diam}(K)\left|\widetilde{F}_{K, \sigma}\right|$ (and u has been extended by 0 outside $\Omega$ ). 
Lemma 6.4 [Compactness property] Assume that (2) holds. Let $\left(\mathcal{D}_{m}\right)_{m \geq 1}$ be admissible discretizations of $\Omega$ in the sense of Definition 2.1, such that $\operatorname{size}\left(\mathcal{D}_{m}\right) \rightarrow 0$ as $m \rightarrow \infty$ and $\left(\operatorname{regul}\left(\mathcal{D}_{m}\right)\right)_{m \geq 1}$ is bounded. Let $\left(\nu_{m}\right)_{m \geq 1}$ be a sequence of nonnegative real numbers and, for all $m \geq 1$, let $\left(u_{m}, \mathbf{v}_{m}, \widetilde{F}_{m}\right)_{m \geq 1}$ satisfy (58) with $\mathcal{D}=\mathcal{D}_{m}$ and $\nu=\nu_{m}$. Assume that the sequence $\left(\mathbf{v}_{m}\right)_{m \geq 1}$ is bounded in $L^{2}(\Omega)^{d}$ and that $N_{2}\left(\mathcal{D}_{m}, \nu_{m}, \widetilde{F}_{m}\right) \rightarrow 0$ as $m \rightarrow \infty$ ( $N_{2}$ has been defined in Lemma 6.2).

Then there exists a subsequence of $\left(\mathcal{D}_{m}\right)_{m \geq 1}$ (still denoted by $\left(\mathcal{D}_{m}\right)_{m \geq 1}$ ) and $\bar{u} \in H_{0}^{1}(\Omega)$ such that the corresponding sequence $\left(u_{m}\right)_{m \geq 1}$ converges to $\bar{u}$ strongly in $L^{q}(\Omega)$ for all $q<\frac{2 d}{d-2}$ (and weakly in $L^{6}(\Omega)$ if $\left.d=3\right)$, and such that $\left(\mathbf{v}_{m}\right)_{m \geq 1}$ converges to $\nabla \bar{u}$ weakly in $L^{2}(\Omega)^{d}$.

\subsection{Tools for the convergence of the nonlinear term}

To study the convergence of the nonlinear term in the scheme for Navier-Stokes problem, it is convenient to introduce a partition of $\Omega$ adapted to the edges of the discretization, and to study the convergence of some special functions defined on such partitions.

Definition 6.1 Assume that (2) holds and let $\mathcal{D}$ be an admissible discretization of $\Omega$ in the sense of Definition 2.1. An $\mathcal{D}$-adapted diamond partition of $\Omega$ is any partition (up to sets of null measure) $\diamond$ of $\Omega$ defined in the following way: for all $K \in \mathcal{M}$, take $\mathbf{p}_{K} \in K$ such that $K$ is star-shaped with respect to all the points in $B\left(\mathbf{p}_{K}, \rho_{K}\right) \beta$ and define $\diamond=\left(\diamond_{\sigma}\right)_{\sigma \in \mathcal{E}}$ as $\diamond_{\sigma}=\operatorname{co}\left(\left\{\mathbf{p}_{K}\right\} \cup \sigma\right) \cup \operatorname{co}\left(\left\{\mathbf{p}_{L}\right\} \cup \sigma\right)$ if $\sigma^{K \mid L} \in \mathcal{E}_{\text {int }}$ and $\diamond_{\sigma}=\operatorname{co}\left(\left\{\mathbf{p}_{K}\right\} \cup \sigma\right)$ if $\sigma^{K \mid \partial} \in \mathcal{E}_{\text {ext }}$ (where $\operatorname{co}(A)$ denotes the convex hull of a set $A)$.

If $w=\left(w_{\sigma}\right)_{\sigma \in \mathcal{E}}$ is a given family of values and $\diamond$ is a $\mathcal{D}$-adapted diamond partition of $\Omega$, the diamond-adapted function defined by $w$ is the piecewise function $w_{\diamond}: \Omega \rightarrow \mathbb{R}$ which is equal, on each $\diamond_{\sigma}$, to $w_{\sigma}$.

Lemma 6.5 Let $T>0$ and assume that (2) holds. Let $\left(\mathcal{D}_{m}\right)_{m \geq 1}$ be a sequence of admissible discretizations of $\Omega$ in the sense of Definition 2.1 such that $\left(\operatorname{regul}\left(\mathcal{D}_{m}\right)\right)_{m \geq 1}$ is bounded and $\operatorname{size}\left(\mathcal{D}_{m}\right) \rightarrow 0$ as $m \rightarrow \infty$. We assume that, for all $m \geq 1$ and all $\delta t$ such that $N_{\delta t}=T / \delta t$ is an integer, we have $u_{m, \delta t} \in H_{\mathcal{D}_{m}, \delta t}$ (see Section 2.3) such that $\sum_{n=0}^{N_{\delta t}-1} \delta t\left\|\left(u_{m, \delta t}\right)^{n+1 / 2}\right\|_{1, \mathcal{D}_{m}}^{2}$ stays bounded as $m \rightarrow \infty$ and $\delta t \rightarrow 0\left(\|\cdot\|_{1, \mathcal{D}_{m}}\right.$ is given by (59) with $\left.\mathcal{D}=\mathcal{D}_{m}\right)$.

For each $m \geq 1$, we choose a $\mathcal{D}_{m}$-adapted diamond partition $\diamond_{m}$. We let, for $n \in\left\{0, \ldots, N_{\delta t}-1\right\}$ and $t \in] n \delta t,(n+1) \delta t\left[,\left(V_{m, \delta t}\right)_{\diamond}(t, \cdot)\right.$ be the (vector-valued) diamond-adapted function defined by the family of (vector) values

$$
\begin{cases}\frac{\mathrm{m}(\sigma)}{\mathrm{m}\left(\diamond_{\sigma}\right)}\left(\left(u_{m, \delta t}\right)_{L}^{n+1 / 2}-\left(u_{m, \delta t}\right)_{K}^{n+1 / 2}\right) \mathbf{n}_{K, \sigma} & \text { if } \sigma^{K \mid L} \in \mathcal{E}_{m, \text { int }} \\ \frac{\mathrm{m}(\sigma)}{\mathrm{m}\left(\diamond_{\sigma}\right)}\left(0-\left(u_{m, \delta t}\right)_{K}^{n+1 / 2}\right) \mathbf{n}_{K, \sigma} & \text { if } \sigma^{K \mid \partial} \in \mathcal{E}_{m, \text { ext }} .\end{cases}
$$

We also assume that $u_{m, \delta t}$ converges to some $\bar{u}$ weakly in $L^{2}(] 0, T[\times \Omega)$ as $m \rightarrow \infty$ and $\delta t \rightarrow 0$. Then $\left(V_{m, \delta t}\right) \diamond$ converges to $\nabla \bar{u}$ weakly in $L^{2}(] 0, T[\times \Omega)^{d}$ as $m \rightarrow \infty$ and $\delta t \rightarrow 0$.

\section{Proof of Lemma 6.5}

\footnotetext{
${ }^{3}$ Recall that $\rho_{K}$ is the supremum of the real numbers $r>0$ such that $K$ is star-shaped with respect to all the points in a ball of radius $r$; it is easy to see that this supremum is a maximum.
} 
To simplify the notations, we drop the indices $m$ and $\delta t$, and we study the convergence of $V_{\diamond}$ as $\operatorname{size}(\mathcal{D}) \rightarrow 0$ and $\delta t \rightarrow 0$ while $\operatorname{regul}(\mathcal{D})$ stays bounded. Let us first show that $V_{\diamond}$ is bounded in $L^{2}(] 0, T[\times \Omega)^{d}$. By definition of $V_{\diamond}$, we have

$$
\begin{aligned}
\left\|V_{\diamond}\right\|_{L^{2}(] 0, T[\times \Omega)^{d}}^{2} & =\sum_{n=0}^{N_{\delta t}-1} \delta t \sum_{\sigma \in \mathcal{E}} \mathrm{m}\left(\diamond_{\sigma}\right)\left|\frac{\mathrm{m}(\sigma)}{\mathrm{m}\left(\diamond_{\sigma}\right)}\left(u_{L}^{n+1 / 2}-u_{K}^{n+1 / 2}\right) \mathbf{n}_{K, \sigma}\right|^{2} \\
& =\sum_{n=0}^{N_{\delta t}-1} \delta t \sum_{\sigma \in \mathcal{E}} \frac{\mathrm{m}(\sigma)}{\mathrm{m}\left(\diamond_{\sigma}\right)} \mathrm{m}(\sigma)\left|u_{L}^{n+1 / 2}-u_{K}^{n+1 / 2}\right|^{2}
\end{aligned}
$$

(we have denoted $\sigma=\sigma^{K \mid L}$ if $\sigma \in \mathcal{E}_{\text {int }}$ and $u_{L}^{n+1 / 2}=0$ if $\sigma=\sigma^{K \mid \partial} \in \mathcal{E}_{\text {ext }}$ ). If $d_{K, \sigma}$ is the orthogonal distance between the $\mathbf{p}_{K}$ chosen to define $\diamond$ and $\sigma \in \mathcal{E}_{K}$ (i.e. the distance between $\mathbf{p}_{K}$ and the hyperplane containing $\left.\sigma\right)$, we have $\mathrm{m}\left(\operatorname{co}\left(\mathbf{p}_{K} \cup \sigma\right)\right)=\mathrm{m}(\sigma) d_{K, \sigma} / d$ and therefore $\mathrm{m}\left(\diamond_{\sigma}\right)=\mathrm{m}(\sigma)\left(d_{K, \sigma}+d_{L, \sigma}\right) / d$ (with $d_{L, \sigma}=0$ if $\left.\sigma=\sigma^{K \mid \partial} \in \mathcal{E}_{\text {ext }}\right)$; hence, $\frac{\mathrm{m}(\sigma)}{\mathrm{m}\left(\diamond_{\sigma}\right)}=\frac{d}{d_{K, \sigma}+d_{L, \sigma}}$. However, since $K$ is star-shaped with respect to all the points in $B\left(\mathbf{p}_{K}, \rho_{K}\right)$, it is possible to show that $d_{K, \sigma} \geq \rho_{K}$ (see the proof of (69) in the second step of the proof of Lemma 6.6); since $\operatorname{regul}(\mathcal{D})$ is bounded, we deduce that there exists $C_{24}$ not depending on $\mathcal{D}$ or $\delta t$ such that

$$
\frac{\mathrm{m}(\sigma)}{\mathrm{m}\left(\diamond_{\sigma}\right)} \leq \frac{C_{24}}{\operatorname{diam}(K)+\operatorname{diam}(L)}
$$

(where $\operatorname{diam}(L)=0$ if $\sigma=\sigma^{K \mid \partial} \in \mathcal{E}_{\text {ext }}$ ) and, coming back to (63),

$$
\|\left. V_{\diamond}\right|_{L^{2}(] 0, T[\times \Omega)^{d}} ^{2} \leq C_{24} \sum_{n=0}^{N_{\delta t}-1} \delta t \sum_{\sigma \in \mathcal{E}} \frac{\mathrm{m}(\sigma)}{\operatorname{diam}(K)+\operatorname{diam}(L)}\left|u_{L}^{n+1 / 2}-u_{K}^{n+1 / 2}\right|^{2} .
$$

As $\frac{1}{\operatorname{diam}(K)+\operatorname{diam}(L)} \leq \frac{1}{\operatorname{diam}(K)}+\frac{1}{\operatorname{diam}(L)}$ for all control volumes $K$ and $L$, we deduce that $\left\|\left.V_{\diamond}\right|_{L^{2}(] 0, T[\times \Omega)^{d}} ^{2} \leq C_{24} \sum_{n=0}^{N_{\delta t}-1} \delta t\right\| u^{n+1 / 2} \|_{1, \mathcal{D}}^{2}$ and, by assumption, that $V_{\diamond}$ is therefore bounded in $L^{2}(] 0, T[\times \Omega)^{d}$.

We now show that $V_{\diamond} \rightarrow \nabla \bar{u}$ in the sense of the distributions on $] 0, T[\times \Omega$ as $\operatorname{size}(\mathcal{D}) \rightarrow 0$ and $\delta t \rightarrow 0$, which concludes the proof of the lemma. Let $\varphi \in C_{c}^{\infty}(] 0, T[\times \Omega)^{d}$ and $\psi(t, \cdot)$ be the function equal to $\frac{1}{\mathrm{~m}(\sigma)} \int_{\sigma} \varphi(t, x) \mathrm{d} \gamma(x)$ on $\diamond_{\sigma}$; since $\varphi$ is regular, we have $\|\varphi-\psi\|_{\infty} \leq C_{25} \operatorname{size}(\mathcal{D})$ with $C_{25}$ only depending on $\varphi$. Hence, $V_{\diamond}$ being bounded in $L^{2}(] 0, T[\times \Omega)^{d}$,

$$
\left|\int_{0}^{T} \int_{\Omega} V_{\diamond}(t, x) \cdot \varphi(t, x) \mathrm{d} t \mathrm{~d} x-\int_{0}^{T} \int_{\Omega} V_{\diamond}(t, x) \cdot \psi(t, x) \mathrm{d} t \mathrm{~d} x\right| \rightarrow 0
$$

as $\operatorname{size}(\mathcal{D}) \rightarrow 0$ and $\delta t \rightarrow 0$. On the other hand, gathering by control volumes,

$$
\begin{aligned}
\int_{0}^{T} \int_{\Omega} V_{\diamond}(t, x) \cdot \psi & (t, x) \mathrm{d} t \mathrm{~d} x \\
& =\sum_{n=0}^{N_{\delta t}-1} \sum_{\sigma^{K \mid L} \in \mathcal{E}_{\text {int }}}\left(u_{L}^{n+1 / 2}-u_{K}^{n+1 / 2}\right) \mathbf{n}_{K, \sigma} \cdot \int_{n \delta t}^{(n+1) \delta t} \int_{\sigma} \varphi(t, x) \mathrm{d} t \mathrm{~d} \gamma(x) \\
& =-\sum_{n=0}^{N_{\delta t}-1} \sum_{K \in \mathcal{M}} u_{K}^{n+1 / 2} \int_{n \delta t}^{(n+1) \delta t} \sum_{\sigma \in \mathcal{E}_{K}} \int_{\sigma} \varphi(t, x) \cdot \mathbf{n}_{K, \sigma} \mathrm{d} t \mathrm{~d} \gamma(x)
\end{aligned}
$$




$$
\begin{aligned}
& =-\sum_{n=0}^{N_{\delta t}-1} \sum_{K \in \mathcal{M}} u_{K}^{n+1 / 2} \int_{n \delta t}^{(n+1) \delta t} \int_{K} \operatorname{div}(\varphi)(t, x) \mathrm{d} t \mathrm{~d} x \\
& =-\int_{0}^{T} \int_{\Omega} u(t, x) \operatorname{div}(\varphi)(t, x) \mathrm{d} t \mathrm{~d} x
\end{aligned}
$$

which converges, by assumption on $u$, to $-\int_{0}^{T} \int_{\Omega} \bar{u}(t, x) \operatorname{div}(\varphi)(t, x) \mathrm{d} t \mathrm{~d} x$. Together with (64), this shows that $V_{\diamond} \rightarrow \nabla \bar{u}$ in the sense of the distributions, and the proof is concluded.

\subsection{A technical result}

The following lemma is the generalization of Lemma 6.3 in [6] to the case of non-convex control volumes.

Lemma 6.6 Let $\alpha>0$ and $d \geq 1$. Assume that $K$ is a polygonal open subset of $\mathbb{R}^{d}$ such that $K$ is star-shaped with respect to all the points in a ball of radius $\alpha \operatorname{diam}(K)$. Let $E$ be an affine hyperplane of $\mathbb{R}^{d}$ and $\sigma$ be a non-empty open subset of $E \cap \partial K$. Then there exists $C_{26}$ only depending on $d$ and $\alpha$ such that, for all $v \in H^{1}(K)$,

$$
\left(\frac{1}{\mathrm{~m}(\sigma)} \int_{\sigma} v(x) \mathrm{d} \gamma(x)-\frac{1}{\mathrm{~m}(K)} \int_{K} v(x) \mathrm{d} x\right)^{2} \leq \frac{C_{26} \operatorname{diam}(K)}{\mathrm{m}(\sigma)} \int_{K}|\nabla v(x)|^{2} d x .
$$

\section{Proof of Lemma 6.6}

In the special case $d=1, K$ is convex and the result is a consequence of Lemma 6.3 in [6]. In the following, we therefore assume $d \geq 2$.

Step 1: a preliminary inequality.

Let $v$ be a regular function and $U, V, A$ be sets in $\mathbb{R}^{d}$ of non-null Lebesgue measure such that, for all $x \in U$ and all $y \in V,[x, y] \subset A$. We prove in this step that there exists $C_{27}$ only depending on $d$ such that

$$
\left(\frac{1}{\mathrm{~m}(U)} \int_{U} v(x) \mathrm{d} x-\frac{1}{\mathrm{~m}(V)} \int_{V} v(x) \mathrm{d} x\right)^{2} \leq \frac{C_{27} \operatorname{diam}(A)^{d+2}}{\mathrm{~m}(U) \mathrm{m}(V)} \int_{A}|\nabla v(x)|^{2} \mathrm{~d} x .
$$

Since $v$ is regular we can write, for all $x \in U$ and all $y \in V, v(x)-v(y)=\int_{0}^{1} \nabla v(t x+(1-t) y)$. $(x-y) \mathrm{d} t$. As $[x, y] \subset A$, we have $|x-y| \leq \operatorname{diam}(A)$ and Jensen's inequality thus implies

$$
\begin{aligned}
& \left(\frac{1}{\mathrm{~m}(U)} \int_{U} v(x) \mathrm{d} x-\frac{1}{\mathrm{~m}(V)} \int_{V} v(x) \mathrm{d} x\right)^{2} \\
& \quad \leq \frac{\operatorname{diam}(A)^{2}}{\mathrm{~m}(U) \mathrm{m}(V)} \int_{U} \int_{V} \int_{0}^{1}|\nabla v(t x+(1-t) y)|^{2} \mathrm{~d} t \mathrm{~d} y \mathrm{~d} x .
\end{aligned}
$$

Let $y \in V$. Using the change of variable $x \in U \rightarrow z=t x+(1-t) y \in A$ and Fubini's theorem, we find

$$
\int_{U} \int_{V} \int_{0}^{1}|\nabla v(t x+(1-t) y)|^{2} \mathrm{~d} t \mathrm{~d} x \mathrm{~d} y \leq \int_{A}|\nabla v(z)|^{2} \int_{V} \int_{I(z, y)} t^{-d} \mathrm{~d} t \mathrm{~d} y \mathrm{~d} z
$$


where $I(z, y)=\{t \in[0,1] \mid \exists x \in U, t x+(1-t) y=z\}$. If $z \in A, y \in V$ and $t \in I(z, y)$, then $t(x-y)=z-y$ for some $x \in U \subset A$, and therefore $\operatorname{diam}(A) t \geq t|x-y|=|z-y|$. Hence $I(z, y) \subset\left[\frac{|z-y|}{\operatorname{diam}(A)}, 1\right]$ and we deduce that

$$
\int_{I(z, y)} t^{-d} \mathrm{~d} t \leq \int_{\frac{|z-y|}{\operatorname{diam}(A)}}^{1} t^{-d} \mathrm{~d} t \leq \frac{1}{d-1} \frac{\operatorname{diam}(A)^{d-1}}{|z-y|^{d-1}} .
$$

Thus, for all $z \in A$,

$$
\int_{V} \int_{I(z, y)} t^{-d} d t d y \leq \frac{\operatorname{diam}(A)^{d-1}}{d-1} \int_{V} \frac{1}{|z-y|^{d-1}} \mathrm{~d} y=\frac{\operatorname{diam}(A)^{d-1}}{d-1} \int_{z-V} \frac{1}{|\xi|^{d-1}} \mathrm{~d} \xi .
$$

Since $V \subset A$, for all $z \in A$ the set $z-V$ is contained in $B(0, \operatorname{diam}(A))$, which allows to write, using polar coordinates,

$$
\int_{V} \int_{I(z, y)} t^{-d} \mathrm{~d} t \mathrm{~d} y \leq \frac{\operatorname{diam}(A)^{d-1}}{d-1} C_{28} \int_{0}^{\operatorname{diam}(A)} \frac{1}{\rho^{d-1}} \rho^{d-1} \mathrm{~d} \rho=\frac{C_{28}}{d-1} \operatorname{diam}(A)^{d}
$$

where $C_{28}$ is the surface of the unit sphere in $\mathbb{R}^{d}$. Substituting this last inequality in (67) and coming back to (66), we conclude the proof of (65).

Step 2: proof of the lemma.

Since the regular functions are dense in $H^{1}(K)$ (because $K$ is star-shaped), it is sufficient to prove the result for $v \in C^{1}(\bar{K})$. Let $\mathbf{p} \in K$ be such that $K$ is star-shaped with respect to all the points in $B(\mathbf{p}, \alpha \operatorname{diam}(K))$.

Let $\triangle$ be the convex hull of $\mathbf{p}$ and $\sigma$ (notice that $\triangle \subset \bar{K}$ ). Under the assumption that $K$ is convex and that $B(\mathbf{p}, \alpha \operatorname{diam}(K)) \subset K$, Lemma 6.2 in [6] states that

$$
\left(\frac{1}{\mathrm{~m}(\sigma)} \int_{\sigma} v(x) \mathrm{d} \gamma(x)-\frac{1}{\mathrm{~m}(\triangle)} \int_{\triangle} v(x) \mathrm{d} x\right)^{2} \leq \frac{C_{29} \operatorname{dist}(\mathbf{p}, E)^{2}}{\mathrm{~m}(\triangle)} \int_{\triangle}|\nabla v(x)|^{2} \mathrm{~d} x
$$

with $C_{29}$ only depending on $d$ and $\alpha$. In fact, in the proof of [6, Lemma 6.2], the convexity assumption on $K$ is only used to ensure that

$$
\operatorname{dist}(\mathbf{p}, E) \geq \alpha \operatorname{diam}(K),
$$

which is a consequence of the fact that $B(\mathbf{p}, \alpha \operatorname{diam}(K))$ entirely lies on one side of $E$; it is quite easy to see that this property still holds if $K$ is only star-shaped with respect to all the points in $B(\mathbf{p}, \alpha \operatorname{diam}(K))$.

Indeed, by translation we can assume that 0 is in the relative interior of $\sigma$. Since $K$ is a polygonal subset, by definition this implies that $K$ is, on a neighborhood of 0 , on one side of its edge $\sigma$; let $\mathbf{n}$ be the outer normal to $K$ on $\sigma$ : we have then $z \cdot \mathbf{n}<0$ for all $z \in K$ in a neighborhood of 0 . Assume now that $B(\mathbf{p}, \alpha \operatorname{diam}(K))$ has points on the two sides of $E$; this means in particular that there exists $y$ in this ball such that $y \cdot \mathbf{n}>0$. Since $K$ is star-shaped with respect to $y$ we have, for all $x \in K$ and all $\lambda \in] 0,1[, z(\lambda, x)=x+\lambda(y-x) \in K$. If $x$ is close to 0 (it is possible to take such a $x$ in $K$ since $0 \in \sigma \subset \partial K)$ and $\lambda$ is close to 0 , we see that $z(\lambda, x)$ is close to 0 ; moreover, $z(\lambda, x) \cdot \mathbf{n}=(1-\lambda) x \cdot \mathbf{n}+\lambda y \cdot \mathbf{n}$ and, since $y \cdot \mathbf{n}>0$, it is possible to choose $x$ close to 0 (so that $x \cdot \mathbf{n}$ is also close to 0 ) and then $\lambda$ close to 0 such that $z(\lambda, x) \cdot \mathbf{n}>0$, which is a 
contradiction since $z(\lambda, x)$ is a point in $K$ close to 0 . Hence, $B(\mathbf{p}, \alpha \operatorname{diam}(K))$ lies on only one side of $E$, and (69) and (68) are therefore valid under our assumptions.

Using (65) with $U=\triangle \backslash \sigma \subset K, V=B(\mathbf{p}, \alpha \operatorname{diam}(K))$ and $A=K$ (since $K$ is star-shaped with respect to $V$ and $U \subset K$, we indeed have $[x, y] \in A$ for all $x \in U$ and all $y \in V$ ), and since $U=\triangle$ up to $\sigma$ which has a null $d$-dimensional Lebesgue measure, we have

$$
\begin{aligned}
\left(\frac{1}{\mathrm{~m}(\triangle)} \int_{\triangle} v(x) \mathrm{d} x-\right. & \left.\frac{1}{\mathrm{~m}(B(\mathbf{p}, \alpha \operatorname{diam}(K)))} \int_{B(\mathbf{p}, \alpha \operatorname{diam}(K))} v(x) \mathrm{d} x\right)^{2} \\
& \leq \frac{C_{27} \operatorname{diam}(K)^{d+2}}{\mathrm{~m}(\triangle) \mathrm{m}(B(\mathbf{p}, \alpha \operatorname{diam}(K)))} \int_{K}|\nabla v(x)|^{2} \mathrm{~d} x
\end{aligned}
$$

Using once more (65), with $U=B(\mathbf{p}, \alpha \operatorname{diam}(K)), V=K$ and $A=K$, we have

$$
\begin{aligned}
\left(\frac{1}{\mathrm{~m}(B(\mathbf{p}, \alpha \operatorname{diam}(K)))} \int_{B(\mathbf{p}, \alpha \operatorname{diam}(K))} v(x) \mathrm{d} x-\frac{1}{\mathrm{~m}(K)} \int_{K} v(x) \mathrm{d} x\right)^{2} \\
\leq \frac{C_{27} \operatorname{diam}(K)^{d+2}}{\mathrm{~m}(B(\mathbf{p}, \alpha \operatorname{diam}(K))) \mathrm{m}(K)} \int_{K}|\nabla v(x)|^{2} \mathrm{~d} x .
\end{aligned}
$$

Gathering (68), (70) and (71), we get $C_{30}$ only depending on $d$ and $\alpha$ so that

$$
\begin{aligned}
& \left(\frac{1}{\mathrm{~m}(\sigma)} \int_{\sigma} v(x) \mathrm{d} \gamma(x)-\frac{1}{\mathrm{~m}(K)} \int_{K} v(x) \mathrm{d} x\right)^{2} \\
& \quad \leq C_{30}\left(\frac{\operatorname{dist}(\mathbf{p}, E)^{2}}{\mathrm{~m}(\triangle)}+\frac{\operatorname{diam}(K)^{2}}{\mathrm{~m}(\triangle)}+\frac{\operatorname{diam}(K)^{2}}{\mathrm{~m}(K)}\right) \int_{K}|\nabla v(x)|^{2} \mathrm{~d} x
\end{aligned}
$$

We have $\mathrm{m}(K) \geq \mathrm{m}(\triangle)=\frac{\operatorname{dist}(\mathbf{p}, E) \mathrm{m}(\sigma)}{d}$, and (69) therefore concludes the proof.

\section{References}

[1] B.F. Armaly, F. Durst, J.C.F. Pereira and B. Schonung, Experimental and theoretical investigation of backward-facing step flow. J. Fluid Mech., 127:473-496, 1983.

[2] S. Boivin, F. Cayré And J.M. Hérard, A finite volume method to solve the NavierStokes equations for incompressible flows on unstructured meshes, Int. J. Therm. Sci., 38, 806-825, 2000.

[3] F. Boyer AND P. FABrie, Eléments d'analyse pour l'étude de quelques modèles d'écoulements de fluides visqueux incompressibles, Mathématiques et Applications (Springer), 52, 405 p., 2006.

[4] C. Chainais-Hillairet and J. Droniou, Convergence analysis of a mixed finite volume scheme for an elliptic-parabolic system modeling miscible fluid flows in porous media. Submitted.

[5] K. Deimling, Nonlinear functional analysis (Springer), 1985. 
[6] J. Droniou and R. Eymard, A mixed finite volume scheme for anisotropic diffusion problems on any grid, Num. Math., 105 (1), 35-71, 2006.

[7] R. Eymard, T. Gallouët and R. Herbin, Finite Volume Methods. In: Handbook of Numerical Analysis, Vol. VII, pp. 713-1020. Edited by P.G. Ciarlet and J.L. Lions (North Holland), 2000.

[8] R. Eymard, R. Herbin and J.C. Latché, On a stabilized colocated Finite Volume scheme for the Stokes problem, M2AN Math. Model. Numer. Anal., 40(3), 501-527, 2006.

[9] Eymard R., Herbin R. and Latché J.C., Convergence analysis of a colocated finite volume scheme for the incompressible Navier-Stokes equations on general 2 or $3 D$ meshes, to appear in SIAM Journal on Numerical Analysis, see also http://hal.ccsd.cnrs.fr/ccsd00004841.

[10] V. Girault And P.-A. Raviart, Finite element methods for the Navier-Stokes equations: Theory and algorithms (Springer), 1986.

[11] R. Glowinski, Numerical Methods for fluids (Part 3), Handbook of Numerical Analysis, Vol. IX. Edited by P.G. Ciarlet and J.L. Lions (North Holland), 2003.

[12] M.D. Gunzburger, Finite element methods for viscous incompressible flows - A guide to theory, practice, and algorithms, Computer Science and Scientific Computing (Academic Press), 1989.

[13] S.R. Mathur and J.Y. Murthy, Pressure boundary conditions for incompressible flow using unstructured meshes, Numer. Heat Transfer, Part B, 32, 283-298, 1997.

[14] F. Nicoud, Conservative High-Order Finite-Difference Schemes for Low-Mach Number Flows, Journal of Computational Physics, 158, 71-97, 2000.

[15] S.V. Patankar, Numerical Heat Transfer and Fluid Flow, Series in Computational Methods in Mechanics and Thermal Sciences, Minkowycz and Sparrow Eds. (Mc Graw Hill), 1980 .

[16] Y. SAAD, Iterative methods for sparse linear systems, Second edition, Society for Industrial and Applied Mathematics, Philadelphia, PA, xviii+528 pp., 2003.

[17] R. Temam, Navier-Stokes Equations - Studies in mathematics and its applications, J.L. Lions, G. Papanicolaou, R.T. Rockafellar Editors (North-Holland), 1977. 